\title{
MODERATE DEVIATIONS FOR A DIFFUSION TYPE PROCESS IN RANDOM ENVIRONMENT
}

\author{
P. CHIGANSKY AND R. LIPTSER
}

\begin{abstract}
Let $\sigma(u), u \in \mathbb{R}$ be an ergodic stationary Markov chain, taking a finite number of values $a_{1}, \ldots, a_{m}$, and $b(u)=g(\sigma(u))$, where $g$ is a bounded and measurable function.

We consider the diffusion type process

$$
d X_{t}^{\varepsilon}=b\left(X_{t}^{\varepsilon} / \varepsilon\right) d t+\varepsilon^{\kappa} \sigma\left(X_{t}^{\varepsilon} / \varepsilon\right) d B_{t}, t \leq T
$$

subject to $X_{0}^{\varepsilon}=x_{0}$, where $\varepsilon$ is a small positive parameter, $B_{t}$ is a Brownian motion, independent of $\sigma$, and $\kappa>0$ is a fixed constant. We show that for $\kappa<1 / 6$, the family $\left\{X_{t}^{\varepsilon}\right\}_{\varepsilon \rightarrow 0}$ satisfies the Large Deviations Principle (LDP) of the Freidlin-Wentzell type with the constant drift $\mathbf{b}$ and the diffusion $\mathbf{a}$, given by

$$
\mathbf{b}=\sum_{i=1}^{m} \frac{g\left(a_{i}\right)}{a_{i}^{2}} \pi_{i} / \sum_{i=1}^{m} \frac{1}{a_{i}^{2}} \pi_{i}, \quad \mathbf{a}=1 / \sum_{i=1}^{m} \frac{1}{a_{i}^{2}} \pi_{i},
$$

where $\left\{\pi_{1}, \ldots, \pi_{m}\right\}$ is the invariant distribution of the chain $\sigma(u)$.
\end{abstract}

\section{INTRODUCTION}

M. Freidlin and R. Sowers, [5], study the LDP for the vector diffusion Markov processes, defined by the Itô equations with respect to Brownian motion $B_{t}$,

$$
X_{t}^{\varepsilon}=x_{0}+\int_{0}^{t} b\left(X_{s}^{\varepsilon} / \varepsilon\right) d s+\varepsilon^{\kappa} \int_{0}^{t} \sigma\left(X_{s}^{\varepsilon} / \varepsilon\right) d B_{s}
$$

where $\varepsilon>0$ is a small parameter and $b(x)$ and $\sigma(x)$ are smooth periodic functions with period 1. The existence of three different LDP regimes, depending on the value of $\kappa$, is shown in [5]:

$$
\kappa \in\left(0, \frac{1}{2}\right), \quad \kappa=\frac{1}{2} \quad \text { and } \quad \kappa \in\left(\frac{1}{2}, \infty\right),
$$

where in the second and the third regimes the rate functions are not of the classic LDP of Freidlin-Wentzell's type. The first regime is characterized by the same rate function as for a diffusion process $\widehat{X}_{t}^{\varepsilon}$ with constant drift and diffusion parameters. Particularly, in the scalar case

$$
\widehat{X}_{t}^{\varepsilon}=x_{0}+\mathbf{b} t+\varepsilon^{\kappa} \sqrt{\mathbf{a}} B_{t},
$$

where $\mathbf{b}=\int_{0}^{1} \frac{b(s) d s}{\sigma^{2}(s)} / \int_{0}^{1} \frac{d s}{\sigma^{2}(s)} \quad$ and $\quad \mathbf{a}=1 / \int_{0}^{1} \frac{1}{\sigma^{2}(s)} d s$. In this context, following the terminology of [6], we shall refer LDP for $\kappa \in\left(0, \frac{1}{2}\right)$ as MDP (Moderate Deviations Principle).

The aim of this note is to extend the MDP to a scalar diffusion in a random environment, namely, when $b(u)=b(\omega, u)$ and $\sigma(u)=\sigma(\omega, u)$ are random processes, independent of the Brownian motion.

The research of P. Chigansky is supported by the Chateaubriand fellowship. 
We assume that $\sigma(u)$ is a stationary and ergodic Markov chain with a finite alphabet

$$
\mathcal{A}=\left\{a_{1}, \ldots, a_{m}\right\}, a_{i} \neq 0,
$$

having right continuous paths with left limits. We assume that

$$
b(u)=g(\sigma(u))
$$

for some bounded measurable function $g(x)$.

We prove the existence of MDP in the random environment for

$$
\kappa \in\left(0, \frac{1}{6}\right) \text { and } \mathbf{b}=\sum_{i=1}^{m} \frac{g\left(a_{i}\right)}{a_{i}^{2}} \pi_{i} / \sum_{i=1}^{m} \frac{1}{a_{i}^{2}} \pi_{i}, \quad \mathbf{a}=1 / \sum_{i=1}^{m} \frac{1}{a_{i}^{2}} \pi_{i},
$$

where $\pi=\left(\pi_{1}, \ldots, \pi_{m}\right)$ is the invariant distribution of $\sigma(u)$.

In [5] the LDP is derived using the Markov property of $X_{t}^{\varepsilon}$ and asymptotic analysis, as $\varepsilon \rightarrow 0$, of the $\log$ moment generating function (hereafter $\lambda(t)$ is a test function):

$$
\lim _{\varepsilon \rightarrow 0} \varepsilon^{2 \kappa} \log \mathrm{E}\left(\exp \left[\frac{1}{\varepsilon^{2 \kappa}} \int_{0}^{T} \lambda(t) d X_{t}^{\varepsilon}\right]\right)
$$

(see, e.g., Ch. 2.3 and Ch. 5.1 in Dembo and Zeitouni, 2], Dupuis and Ellis, 3], Gartner and Freidlin, 44). When the environment is random, $X_{t}^{\varepsilon}$ is not a Markov process anymore and calculation of the limit (1.3) is quite involved. Instead of (1.3), we apply the Puhalskii approach based on the martingale exponential

$$
\mathfrak{z}_{T}(\lambda)=\exp \left[\frac{1}{\varepsilon^{2 \kappa}} \int_{0}^{T} \lambda(t) d X_{t}^{\varepsilon}-\log \mathscr{E}_{T}\left(X^{\varepsilon}\right)\right]
$$

analysis, where

$$
\mathscr{E}_{t}\left(X^{\varepsilon}\right)=\exp \left(\frac{1}{\varepsilon^{2 \kappa}} \int_{0}^{t}\left[\lambda(s) b\left(X_{s}^{\varepsilon} / \varepsilon\right)-\frac{\lambda^{2}(s)}{2} \sigma^{2}\left(X_{s}^{\varepsilon} / \varepsilon\right)\right] d s\right)
$$

is the cumulant process compensating $\exp \left(\frac{1}{\varepsilon^{2 \kappa}} \int_{0}^{T} \lambda(t) d X_{t}^{\varepsilon}\right)$ up to a local martingale. Sufficient conditions for the MDP can be formulated in terms of the cumulant process (see Corollary 4.3.8 in [13]) and in our setting even directly in terms of the drift and diffusion parameters: by Corollary 6.7 in 14 the MDP holds if for any $\eta>0$ one can choose $\mathbf{b}, \mathbf{a}>0$ and $\kappa>0$ such that

$$
\begin{aligned}
& \lim _{\varepsilon \rightarrow 0} \varepsilon^{2 \kappa} \log \mathrm{P}\left(\sup _{t \leq T}\left|\int_{0}^{t}\left[b\left(X_{s}^{\varepsilon} / \varepsilon\right)-\mathbf{b}\right] d s\right|>\eta\right)=-\infty \\
& \lim _{\varepsilon \rightarrow 0} \varepsilon^{2 \kappa} \log \mathrm{P}\left(\sup _{t \leq T}\left|\int_{0}^{t}\left[\sigma^{2}\left(X_{s}^{\varepsilon} / \varepsilon\right)-\mathbf{a}\right] d s\right|>\eta\right)=-\infty ;
\end{aligned}
$$

the appropriate choice is announced in (1.2)

The next section discusses the weak solutions of (1.1) in the random environment. Our main result, Theorem 3.1, is formulated in Section 3 and is proved in Section 5 which is preceded by auxiliary results in Section 4. The MDP gap between

$$
\kappa \in\left(0, \frac{1}{2}\right) \text { and } \kappa \in\left(0, \frac{1}{6}\right)
$$

for oscillating and random environments is discussed in Section 6 , 
For reader's fast reference, the essential details of Puhalskii's method, adapted to our setting, are outlined in Appendix.

\section{DifFUSION IN RANDOM ENVIRONMENT}

Hereafter, we will deal with a weak solution of the scalar equation explicitly constructed by time scaling and change of probability measure (for other approaches see [1, [15]).

Let $\sigma=(\sigma(u))_{u \in \mathbb{R}}$ be the Markov chain, defined in the previous section, and $\beta=\left(\beta_{t}\right)_{t \geq 0}$ be a Brownian motion independent of $\sigma$. Assume that the pair $(\sigma, \beta)$ is defined on a stochastic basis $\left(\Omega, \mathcal{F}, \mathbf{F}=\left(\mathcal{F}_{t}\right)_{t \geq 0}, \mathrm{Q}\right)$ with the general conditions, $\sigma$ is $\mathcal{F}_{0}$-measurable and $\beta$ is independent of $\mathcal{F}_{0}$.

For $t>0$, introduce the stopping time $\tau_{t}=\inf \left\{r: \int_{0}^{r} \frac{1}{\varepsilon^{2 \kappa} \sigma^{2}\left(\left(\beta_{s}+x_{0}\right) / \varepsilon\right)} d s \geq t\right\}$. Since $\sigma^{2}(u)>0$, we have $\int_{0}^{\tau_{t}} \frac{1}{\varepsilon^{2 \kappa} \sigma^{2}\left(\left(\beta_{s}+x_{0}\right) / \varepsilon\right)} d s \equiv t$ and, in turn,

$$
\tau_{t}=\int_{0}^{t} \varepsilon^{2 \kappa} \sigma^{2}\left(\left(\beta_{\tau_{s}}+x_{0}\right) / \varepsilon\right) d s
$$

Introduce the filtration $\mathbf{G}=\left(\mathcal{G}_{t}\right)_{t \geq 0}$ with $\mathcal{G}_{t}:=\mathcal{F}_{\tau_{t}}$. Obviously, $\left(\beta_{\tau_{t}}, \mathcal{G}_{t}\right)$ is a continuous martingale with the quadratic variation process $\tau_{t}$. Then by the Levy-Doob theorem the process

$$
B_{t}=\int_{0}^{t} \frac{1}{\varepsilon^{\kappa} \sigma\left(\left(\beta_{\tau_{s}}+x_{0}\right) / \varepsilon\right)} d \beta_{\tau_{t}}
$$

is Brownian motion. Since $B=\left(B_{t}\right)_{t>0}$ is independent of $\mathcal{F}_{0}$ and $\mathcal{F}_{0}=\mathcal{G}_{0}, B=\left(B_{t}\right)_{t>0}$ is independent of $\mathcal{G}_{0}$. On the other hand, by the same reason $\sigma$ is $\mathcal{G}_{0}$-measurable. Hence, $\left(B_{t}\right)$ and $\sigma(u)$ are independent random processes.

Denote $Y_{t}:=x_{0}+\beta_{\tau_{t}}$. Then, the definition of $B_{t}$ implies the following representation for $Y_{t}$ :

$$
Y_{t}=x_{0}+\int_{0}^{t} \varepsilon^{\kappa} \sigma\left(Y_{s} / \varepsilon\right) d B_{s}
$$

Consequently, at least one weak solution of (1.1) with zero drift exists. A weak solution of (1.1) with the required drift can be constructed with the help of Girsanov's theorem. With $Y_{t}$, defined in (2.1), set

$$
\Upsilon_{T}=\exp \left(\int_{0}^{T} \frac{b\left(Y_{s} / \varepsilon\right)}{\varepsilon^{\kappa} \sigma\left(Y_{s} / \varepsilon\right)} d B_{s}-\frac{1}{2} \int_{0}^{T} \frac{b^{2}\left(Y_{s} / \varepsilon\right)}{\varepsilon^{2 \kappa} \sigma^{2}\left(Y_{s} / \varepsilon\right)} d s\right)
$$

Since $\frac{b\left(Y_{s} / \varepsilon\right)}{\varepsilon^{\kappa} \sigma\left(Y_{s} / \varepsilon\right)}$ is bounded and $T<\infty$, we have $\int_{\Omega} \Upsilon_{T} d \mathbf{Q}=1$. We define a probability measure $\mathrm{P}$ with $d \mathrm{P}:=\mathfrak{z}_{T} d \mathrm{Q}$. Then, by the Girsanov theorem,

$$
\widehat{B}_{t}=B_{t}-\int_{0}^{t} \frac{b\left(Y_{s} / \varepsilon\right)}{\varepsilon^{\kappa} \sigma\left(Y_{s} / \varepsilon\right)} d s
$$

is the Brownian motion with respect to $\mathbf{G}$ under $\mathrm{P}$. In other words, the process $Y_{t}$ defined on the new stochastic basis $\left(\Omega, \mathcal{F}, \mathbf{G}=\left(\mathcal{G}_{t}\right)_{t \geq 0}, \mathrm{P}\right)$ admits the following representation

$$
Y_{t}=x_{0}+\int_{0}^{t} b\left(Y_{s} / \varepsilon\right) d s+\int_{0}^{t} \varepsilon^{\kappa} \sigma\left(Y_{s} / \varepsilon\right) d \widehat{B}_{s},
$$


that is, $Y_{t}$ is a weak solution of (1.1). Since $\left(\widehat{B}_{t}\right)_{t \leq T}$ is P-independent of $\mathcal{G}_{0}$ and $(\sigma(u))_{\mathbb{R}}$ is $\mathcal{G}_{0}$-measurable, the Brownian motion $\widehat{B}_{t}$ and $\sigma(u)$ are P-independent random processes.

\section{The MAIN RESULT}

Let $X^{\varepsilon}=\left(X_{t}^{\varepsilon}\right)_{t \leq T}$ be a weak solution of (1.1). Recall that $X^{\varepsilon}$ satisfies LDP (in our case MDP) with the rate $\varepsilon^{2 \kappa}$ and the good rate function $J(u)$ in the space of continuous functions $\mathbb{C}_{[0, T]}$ endowed with the uniform metric if for any closed set $F$ and open set $G$,

$$
\begin{aligned}
& \varlimsup_{\varepsilon \rightarrow 0} \varepsilon^{2 \kappa} \log \mathrm{P}\left(X^{\varepsilon} \in F\right) \leq-\inf _{u \in F} J(u) \\
& \varliminf_{\varepsilon \rightarrow 0} \varepsilon^{2 \kappa} \log \mathrm{P}\left(X^{\varepsilon} \in G\right) \geq-\inf _{u \in G} J(u) .
\end{aligned}
$$

Theorem 3.1. For $\kappa<1 / 6$, the family $\left\{X^{\varepsilon}\right\}_{\varepsilon \rightarrow 0}$ satisfies the $M D P$ with the rate $\varepsilon^{2 \kappa}$ and the rate function

$$
J(u)= \begin{cases}\frac{1}{2 \mathbf{a}} \int_{0}^{T}\left[\dot{u}_{t}-\mathbf{b}\right]^{2} d t, & \begin{array}{l}
u_{0}=x_{0} \\
d u_{t}=\dot{u}_{t} d t
\end{array} \\
\infty, & \int_{0}^{T} \dot{u}_{t}^{2} d t<\infty \\
\infty, & \text { otherwise }\end{cases}
$$

with

$$
\mathbf{b}=\sum_{i=1}^{m} \frac{g\left(a_{i}\right) \pi_{i}}{a_{i}^{2}} / \sum_{i=1}^{m} \frac{\pi_{i}}{a_{i}^{2}} \quad \text { and } \quad \mathbf{a}=1 / \sum_{i=1}^{m} \frac{\pi_{i}}{a_{i}^{2}},
$$

where $\left\{\pi_{1}, \ldots, \pi_{m}\right\}$ is the invariant distribution of $\sigma$.

The proof of this theorem requires some auxiliary results gathered in the next section.

\section{Auxiliary Results}

Henceforth,

- $A^{*}$ is transposed of a matrix $A$;

- for any $x \in \mathbb{R}^{d}, \operatorname{diag}(x)$ is the diagonal matrix with (diagonal) entries $x_{i}$ 's;

$-l$ is a generic positive constant whose meaning may change from line to line;

$-\inf \{\varnothing\}=\infty$.

4.1. The Poisson decomposition. Let $\mathbf{F}^{\sigma}=\left(\mathcal{F}_{t}^{\sigma}\right)_{t \in \mathbb{R}}$ be the filtration generated by $\sigma$ : $\mathcal{F}_{t}^{\sigma}=\{\sigma(u),-\infty<u \leq t\}$. Since $\sigma$ is an ergodic chain, its transition intensities matrix $\Lambda$ has simple zero eigenvalue. Therefore, for any bounded measurable function $\Psi(x)$ with $\mathrm{E} \Psi(\sigma(0))=0$ there exists $\gamma>0$ such that $\left|\mathrm{E}\left(\Psi(\sigma(t)) \mid \mathcal{F}_{0}^{\sigma}\right)\right| \leq l e^{-\gamma t}$ a.s. for any $t>0$. Hence, $\int_{0}^{\infty} \mathrm{E} \mid \mathrm{E}\left(\Psi\left(\sigma(t) \mid \mathcal{F}_{0}^{\sigma}\right) \mid d t<\infty\right.$. Then (see e.g. Ch $9, \S 2$ in [10]) the process $\int_{0}^{t} \Psi(\sigma(s)) d s$ obeys the Poisson decomposition

$$
\int_{0}^{t} \Psi(\sigma(s)) d s=V_{t}-V_{0}-M_{t}
$$

where $V_{t}$ is $\mathbf{F}^{\sigma}$-adapted process and $M_{t}$ is $\mathbf{F}^{\sigma}$-martingale with right continuous pathes having left limits. In the case under consideration, $M_{t}$ is a square integrable martingale (see Lemma 4.1) with the quadratic variation process $\langle M\rangle_{t}$. 


\section{Lemma 4.1.}

1) $\left|V_{t}\right| \leq l$ for any $t \geq 0$

2) $M_{t}$ is a purely discontinuous square integrable martingale with bounded jumps;

3) $d\langle M\rangle_{t}=m(t) d t, m(t) \leq l$.

Proof. Denote by

$$
I(t)=\left(\begin{array}{c}
I_{\left\{\sigma(t)=a_{1}\right\}} \\
\vdots \\
I_{\left\{\sigma(t)=a_{m}\right\}}
\end{array}\right) \quad \text { and } \quad f=\left(\begin{array}{c}
\Psi\left(a_{1}\right) \\
\vdots \\
\Psi\left(a_{m}\right)
\end{array}\right) .
$$

The obvious equality $\Psi(\sigma(t))=f^{*} I(t)$ implies

$$
0=\mathrm{E} \Psi(\sigma(t))=f^{*} \mathrm{E} I(t)=f^{*} \pi .
$$

This property of $f$ and the aforementioned spectral gap of the matrix $\Lambda$ guarantees solvability of the Poisson equation

$$
\Lambda g=f
$$

whose solution is unique in the class $g^{*} \pi=0$. Only this solution will be considered in the sequel.

By Lemma 9.2, Ch.9, §9.1 in [9],

$$
N_{t}:=I(t)-I(0)-\int_{0}^{t} \Lambda^{*} I(s) d s
$$

is a purely discontinuous martingale, with respect to $\mathbf{F}^{\sigma}$, with bounded jumps. We show now that $V_{t}=g^{*} I(t)$ and $M_{t}=g^{*} N_{t}$. Multiplying from the left both sides of (4.3) by $g^{*}$ and taking into account the definition of $V_{t}$ and $M_{t}$ we find that $M_{t}=V_{t}-V_{0}-\int_{0}^{t} g^{*} \Lambda^{*} I(s) d s$. Further, by (4.2),$g^{*} \Lambda^{*} I(s)=f^{*} I(s)=\Psi(\sigma(u))$. In other words, (4.1) holds true with $V_{t}$ and $M_{t}$ chosen above. Therefore, statements 1) and 2) are obvious. The statement 3) is proved as follows: by the Itô formula we find that

$$
\begin{aligned}
I(t) I^{*}(t)= & I(0) I^{*}(0)+\int_{0}^{t}\left[I(s) I^{*}(s) \Lambda+\Lambda^{*} I(s) I^{*}(s)\right] d s \\
& +\int_{0}^{t}\left[I(s-) d N_{s}^{*}+d N_{s} I^{*}(s-)\right]+[N, N]_{t} \\
= & I(0) I^{*}(0)+\int_{0}^{t}\left[I(s) I^{*}(s) \Lambda+\Lambda^{*} I(s) I^{*}(s)\right] d s+\langle N\rangle_{t} \\
& + \text { martingale, }
\end{aligned}
$$

where $\langle N\rangle_{t}$ is the quadratic variation process of $N_{t}$, and, owing to the identity $I(t) I^{*}(t)=$ $\operatorname{diag}(I(t))$, also that

$$
I(t) I^{*}(t)=I(0) I^{*}(0)+\int_{0}^{t} \operatorname{diag}\left(\Lambda^{*} I(s)\right) d s+\text { martingale. }
$$

Both representations for $I(t) I^{*}(t)$ imply that the predictable process with paths in the Skorokhod space of locally bounded variation

$$
\int_{0}^{t}\left(\left[I(s) I^{*}(s) \Lambda+\Lambda^{*} I(s) I^{*}(s)\right]-\operatorname{diag}\left[\Lambda^{*} I(s)\right]\right) d s+\langle N\rangle_{t}
$$


is a martingale starting from zero. Hence, by Theorem 1 in Ch. 2, $\S 2$, [10, this martingale is indistinguishable from zero or, equivalently,

$$
\langle N\rangle_{t}=\int_{0}^{t}\left(\operatorname{diag}\left[\Lambda^{*} I(s)\right]-\left[I(s) I^{*}(s) \Lambda+\Lambda^{*} I(s) I^{*}(s)\right]\right) d s .
$$

Therefore, $d\langle M\rangle_{t} \equiv g^{*} d\langle N\rangle_{t} g=g^{*}\left(\operatorname{diag}\left[\Lambda^{*} I(t)\right]-\left[I(t) I^{*}(t) \Lambda+\Lambda^{*} I(t) I^{*}(t)\right]\right) g d t$.

4.2. Exponential estimate for martingales with bounded jumps. For a continuous martingale $M=\left(M_{t}\right)_{t \geq 0}$ with $M_{0}=0$ and the quadratic variation process $\langle M\rangle_{t}$ the following exponential estimate is well known (see e.g. Lemma 1 in [11]): for any $q, r>0$,

$$
\mathrm{P}\left(\sup _{t \leq T}\left|M_{t}\right| \geq r,\langle M\rangle_{T} \leq q\right) \leq 2 \exp \left(-\frac{r^{2}}{2 q}\right) .
$$

A similar inequality holds for discontinuous martingales.

Lemma 4.2. Let $M=\left(M_{t}\right)_{t \geq 0}$ be a purely discontinuous martingale with $M_{0}=0$ and paths in the Skorokhod space $\mathbb{D}$ with bounded jumps $\left|\triangle M_{t}\right| \leq K$ and the quadratic variation process $\langle M\rangle_{t}$. Then, for any $q, r>0$

$$
\mathrm{P}\left(\sup _{t \leq T}\left|M_{t}\right| \geq r,\langle M\rangle_{T} \leq q\right) \leq 2 \exp \left(-\frac{r^{2}}{2(K r+q)}\right) .
$$

Remark 1. (4.4) is a particular case of (4.5) for $K=0$.

Proof. Denote by $\mu=\mu(d t, d z)$ the integer-valued measure, associated with the jump process $\triangle M_{t}$, and by $\nu=\nu(d t, d z)$ the compensator of $\mu$ (for more details, see e.g. [10] or [8]); clearly $\nu\left(\mathbb{R}_{+} \times\{|z|>K\}\right)=0$ since $\left|\triangle M_{t}\right| \leq K$.

Then $M_{t}=\int_{0}^{t} \int_{|z| \leq K} z[\mu(d s, d z)-\nu(d s, d z)]$ and $\langle M\rangle_{t}=\int_{0}^{t} \int_{|z| \leq K} z^{2} \nu(d s, d z)$. Let $\mathscr{L}_{t}(\lambda)$ be the cumulant process associated with $M_{t}$, i.e. for any $\lambda \in \mathbb{R}$ the random process

$$
\mathfrak{z}_{t}(\lambda)=e^{\lambda M_{t}-\mathscr{L}_{t}(\lambda)}
$$

is a local martingale. It can be easily checked with the help of Itô's formula that

$$
\mathscr{L}_{t}(\lambda)=G_{t}(\lambda)+\sum_{s \leq t}\left[\log \left(1+\triangle G_{s}(\lambda)\right)-\triangle G_{s}(\lambda)\right]
$$

where

$$
\begin{aligned}
G_{t}(\lambda) & =\int_{0}^{t} \int_{|z| \leq K}\left(e^{\lambda z}-1-\lambda z\right) \nu(d s, d z) \\
\triangle G_{t}(\lambda) & =\int_{|z| \leq K}\left(e^{\lambda z}-1-\lambda z\right) \nu(\{t\}, d z) .
\end{aligned}
$$

The positive local martingale $\mathfrak{z}_{t}(\lambda)$ is also a supermartingale. Hence $\mathrm{E}_{\mathfrak{z} \tau}(\lambda) \leq 1$ for any stopping time $\tau$. Since $\triangle G_{t}(\lambda) \geq 0$ and $\log (1+x)-x \leq 0$ for $x \geq 0$, we have $\mathscr{L}_{t}(\lambda) \leq G_{t}(\lambda)$. Consequently, $\mathfrak{z}_{\tau} \geq e^{\lambda M_{\tau}-G_{\tau}(\lambda)}$ for any stopping time $\tau$, that is,

$$
\mathrm{E} e^{\lambda M_{\tau}-G_{\tau}(\lambda)} \leq 1
$$


For $|z| \leq K$ and $|\lambda|<1 / K$, we have

$$
e^{\lambda z}-\lambda z-1=\sum_{j=2}^{\infty} \frac{(\lambda z)^{j}}{j !} \leq \frac{\lambda^{2} z^{2}}{2} \sum_{j=0}^{\infty}|\lambda z|^{j}=\frac{\lambda^{2} z^{2}}{2} \frac{1}{1-|\lambda z|} \leq \frac{\lambda^{2} z^{2}}{2} \frac{1}{1-|\lambda K|} .
$$

Hence

$$
G_{\tau}(\lambda) \leq \int_{0}^{\tau} \int_{|z| \leq K} \frac{\lambda^{2} z^{2}}{2} \frac{1}{1-|\lambda K|} \nu(d s, d z)=\frac{1}{1-|\lambda| K} \frac{\lambda^{2}}{2}\langle M\rangle_{\tau} .
$$

Now, due to (4.6), for any measurable set $A$ we obtain that

$$
1 \geq \mathrm{E} I_{\{A\}} \exp \left(\lambda M_{\tau}-\frac{1}{1-|\lambda| K} \frac{\lambda^{2}}{2}\langle M\rangle_{\tau}\right)
$$

The choice of $\tau=\inf \left\{t \leq T: M_{t} \geq r\right\}$ and $A=\{\tau \leq T\} \cap\left\{\langle M\rangle_{T} \leq q\right\}$ for any $|\lambda| \leq 1 / K$ implies

$$
1 \geq \mathrm{E} I_{\{A\}} \exp \left(\lambda M_{\tau}-\frac{1}{1-|\lambda| K} \frac{\lambda^{2}}{2}\langle M\rangle_{\tau}\right) \geq \mathrm{E} I_{\{A\}} \exp \left(\lambda r-\frac{1}{1-|\lambda| K} \frac{\lambda^{2}}{2} q\right) .
$$

So, taking into account that $\left\{\sup _{t \leq T} M_{t} \geq r\right\}=\{\tau \leq T\}$, we find that

$$
\mathrm{P}\left(\sup _{t \leq T} M_{t} \geq r,\langle M\rangle_{T} \leq q\right) \leq \exp \left(-\frac{\lambda r(1-\lambda K)-\frac{\lambda^{2}}{2} q}{1-\lambda K}\right) .
$$

Finally, the choice of $\lambda^{\prime}=\underset{\lambda \in\left(0, \frac{1}{K}\right)}{\operatorname{argmax}}\left[\lambda r(1-\lambda K)-\frac{\lambda^{2}}{2} q\right]$ provides

$$
\mathrm{P}\left(\sup _{t \leq T} M_{t} \geq r,\langle M\rangle_{T} \leq q\right) \leq \exp \left(-\frac{r^{2}}{2(K r+q)}\right)
$$

The same inequality holds for $\sup _{t \leq T}\left(-M_{t}\right)$.

Now, (4.5) follows from $\mathrm{P}(A \cup B) \leq 2[\mathrm{P}(A) \vee \mathrm{P}(B)]$, for any measurable sets $A$ and $B$.

\section{The proof of Theorem 3.1}

Recall that (1.4) implies the required MDP. We begin with the proof of the first part in (1.4). Introduce the stationary process

$$
\theta(t)=\frac{b(t)-\mathbf{b}}{\sigma^{2}(t)}
$$

where $\mathbf{b}$ is a fixed constant such that $\mathrm{E} \theta(t)=0$; in other words, $\mathbf{b}=\sum_{i=1}^{m} \frac{g\left(a_{i}\right) \pi_{i}}{a_{i}^{2}} / \sum_{i=1}^{m} \frac{\pi_{i}}{a_{i}^{2}}$.

Define

$$
H(x)=\int_{0}^{x} \int_{0}^{v} \theta(s) d s d v .
$$

The random function $H(x)$ is continuously differentiable and has bounded Sobolev's second derivative. Hence by Krylov's version of the Itô formula [7]

$$
H\left(X_{t}^{\varepsilon} / \varepsilon\right)=H\left(x_{0} / \varepsilon\right)+\frac{1}{\varepsilon} \int_{0}^{t} \int_{0}^{X_{v}^{\varepsilon} / \varepsilon} \theta(s) d s d X_{v}^{\varepsilon}+\frac{1}{\varepsilon^{2(1-\kappa)}} \int_{0}^{t}\left[b\left(X_{s}^{\varepsilon} / \varepsilon\right)-\mathbf{b}\right] d s
$$


or, equivalently,

$$
\begin{array}{r}
\int_{0}^{t}\left[b\left(X_{s}^{\varepsilon} / \varepsilon\right)-\mathbf{b}\right] d s=\varepsilon^{2(1-\kappa)} \int_{0}^{X_{t}^{\varepsilon} / \varepsilon} \int_{0}^{v} \theta(s) d s d v-\varepsilon^{2(1-\kappa)} \int_{0}^{x_{0} / \varepsilon} \int_{0}^{v} \theta(s) d s d v- \\
\varepsilon^{1-2 \kappa} \int_{0}^{t} \int_{0}^{X_{v}^{\varepsilon} / \varepsilon} \theta(s) d s b\left(X_{v}^{\varepsilon} / \varepsilon\right) d v-\varepsilon^{1-\kappa} \int_{0}^{t} \int_{0}^{X_{v}^{\varepsilon} / \varepsilon} \theta(s) d s \sigma\left(X_{v}^{\varepsilon} / \varepsilon\right) d B_{v} .
\end{array}
$$

Let $Z_{t}^{\varepsilon}$ denote any of the terms in the right hand side of (5.1). Obviously, the first part (1.4) holds true if

$$
\lim _{\varepsilon \rightarrow 0} \varepsilon^{2 \kappa} \log \mathrm{P}\left(\sup _{t \leq T}\left|Z_{t}^{\varepsilon}\right|>\eta\right)=-\infty .
$$

In order to simplify the proof of (15.2), let us show that the set $\Upsilon_{C}^{\varepsilon}=\left\{\sup _{t \leq T}\left|X_{t}^{\varepsilon}\right|>C\right\}$ is exponentially negligible in the sense

$$
\lim _{C \rightarrow \infty} \varlimsup_{\varepsilon \rightarrow 0} \varepsilon^{2 \kappa} \log \mathrm{P}\left(\Upsilon_{C}^{\varepsilon}\right)=-\infty .
$$

Denote by $A_{t}^{\varepsilon}=x_{0}+\int_{0}^{t} b\left(X_{s}^{\varepsilon} / \varepsilon\right) d s$ and $M_{t}^{\varepsilon}=\varepsilon^{\kappa} \int_{0}^{t} \sigma\left(X_{s}^{\varepsilon} / \varepsilon\right) d B_{s}$. Since $X_{t}^{\varepsilon}=A_{t}^{\varepsilon}+M_{t}^{\varepsilon}$ and $\sup _{t \leq T}\left|A_{t}^{\varepsilon}\right|$ is bounded by a constant independent of $\varepsilon$, the proof of (5.3) reduces to the proof of

$$
\lim _{C \rightarrow \infty} \varlimsup_{\varepsilon \rightarrow 0} \varepsilon^{2 \kappa} \log \mathrm{P}\left(\sup _{t \leq T}\left|M_{t}^{\varepsilon}\right|>C\right)=-\infty .
$$

Since $\mathrm{P}\left(\left\langle M^{\varepsilon}\right\rangle_{T} \leq l T \varepsilon^{2 \kappa}\right)=1$, due to (4.4), we have $\mathrm{P}\left(\sup _{t \leq T}\left|M_{t}^{\varepsilon}\right|>C\right) \leq 2 \exp \left(-\frac{C^{2}}{2 \varepsilon^{2 \kappa} l T}\right)$ and in turn (5.3).

In view of (5.3), instead of (5.2) it suffices to prove that for any $C>0$

$$
\varlimsup_{\varepsilon \rightarrow \infty} \varepsilon^{2 \kappa} \log \mathrm{P}\left(\sup _{t \leq T}\left|Z_{t}^{\varepsilon}\right| \geq \eta, \Omega \backslash \Upsilon_{C}^{\varepsilon}\right)=-\infty .
$$

Let $Z_{t}^{\varepsilon}:=\varepsilon^{2(1-\kappa)} \int_{0}^{X_{t}^{\varepsilon} / \varepsilon} \int_{0}^{v} \theta(s) d s d v$. Then, $\sup _{t \leq T}\left|Z_{t}^{\varepsilon}\right|$ is bounded from above on the set $\left\{\Omega \backslash \Upsilon_{C}^{\varepsilon}\right\}$ by $C \sup _{|v| \leq C}\left|\varepsilon^{1-2 \kappa} \int_{0}^{v / \varepsilon} \theta(s) d s\right|$. Consequently,

$$
\begin{aligned}
\mathrm{P}\left(\sup _{t \leq T}\left|Z_{t}^{\varepsilon}\right| \geq \eta, \Omega \backslash \Upsilon_{C}^{\varepsilon}\right) & \leq \mathrm{P}\left(\sup _{|v| \leq C}\left|\varepsilon^{1-2 \kappa} \int_{0}^{v / \varepsilon} \theta(s) d s\right| \geq \eta, \Omega \backslash \Upsilon_{C}^{\varepsilon}\right) \\
& \leq \mathrm{P}\left(\sup _{|v| \leq C}\left|\varepsilon^{1-2 \kappa} \int_{0}^{v / \varepsilon} \theta(s) d s\right| \geq \eta\right)
\end{aligned}
$$

and (5.4) holds provided that

$$
\lim _{\varepsilon \rightarrow 0} \varepsilon^{2 \kappa} \log \mathrm{P}\left(\sup _{|v| \leq C}\left|\varepsilon^{1-2 \kappa} \int_{0}^{v / \varepsilon} \theta(s) d s\right| \geq \eta\right)=-\infty .
$$

For $Z_{t}^{\varepsilon}:=\varepsilon^{1-2 \kappa} \int_{0}^{t} \int_{0}^{X_{v}^{\varepsilon} / \varepsilon} \theta(s) d s b\left(X_{v}^{\varepsilon} / \varepsilon\right) d v$ and $Z_{t}^{\varepsilon}:=\varepsilon^{2(1-\kappa)} \int_{0}^{x_{0} / \varepsilon} \int_{0}^{v} \theta(s) d s d v$, (5.5) implies (5.4).

Recall that $\theta(s)$ is a strictly stationary process and, therefore, the distributions of

$$
\sup _{0 \leq v \leq C}\left|\int_{0}^{v / \varepsilon} \theta(s) d s\right| \text { and } \sup _{0 \leq v \leq C}\left|\int_{-v / \varepsilon}^{0} \theta(s) d s\right|
$$


coincide. Hence,

$$
\begin{aligned}
& \mathrm{P}\left(\sup _{|v| \leq C}\left|\varepsilon^{1-2 \kappa} \int_{0}^{v / \varepsilon} \theta(s) d s\right| \geq \eta\right) \\
& \leq 2\left[\mathrm{P}\left(\sup _{0 \leq v \leq C}\left|\varepsilon^{1-2 \kappa} \int_{0}^{v / \varepsilon} \theta(s) d s\right| \geq \frac{\eta}{2}\right) \bigvee \mathrm{P}\left(\sup _{0 \leq v \leq C}\left|\varepsilon^{1-2 \kappa} \int_{-v / \varepsilon}^{0} \theta(s) d s\right| \geq \frac{\eta}{2}\right)\right] \\
& =2 \mathrm{P}\left(\sup _{0 \leq v \leq C}\left|\varepsilon^{1-2 \kappa} \int_{0}^{v / \varepsilon} \theta(s) d s\right| \geq \frac{\eta}{2}\right)
\end{aligned}
$$

Thus, instead of (5.5), we shall prove

$$
\lim _{\varepsilon \rightarrow 0} \varepsilon^{2 \kappa} \log \mathrm{P}\left(\sup _{0<v \leq C}\left|\varepsilon^{1-2 \kappa} \int_{0}^{v / \varepsilon} \theta(s) d s\right| \geq \frac{\eta}{2}\right)=-\infty .
$$

Since $\theta(s)=\Psi(\sigma(s))$ and $\mathrm{E} \theta(s)=0$, by Lemma 4.1 we have

$$
\varepsilon^{1-2 \kappa} \int_{0}^{v / \varepsilon} \theta(s) d s=\varepsilon^{1-2 \kappa}\left[V_{v / \varepsilon}-V_{0}\right]+\varepsilon^{1-2 \kappa} M_{v / \varepsilon},
$$

where $V_{t}$ is a bounded process and $M_{t}$ is a purely discontinuous martingale with bounded jumps $\left|\triangle M_{s}\right| \leq l$ and $\langle M\rangle_{v} \leq l v$. Therefore, we shall prove only that for any $\eta>0$,

$$
\lim _{\varepsilon \rightarrow 0} \varepsilon^{2 \kappa} \log \mathrm{P}\left(\sup _{0<v \leq C}\left|\varepsilon^{1-2 \kappa} M_{v / \varepsilon}\right| \geq \eta\right)=-\infty
$$

By (4.5) and $\mathrm{P}\left(\left\langle\varepsilon^{1-2 \kappa} M\right\rangle_{C / \varepsilon} \leq l C \varepsilon^{1-4 \kappa}\right)=1$, we have the following upper bound for $\kappa<\frac{1}{4}:$

$$
\mathrm{P}\left(\sup _{0 \leq v \leq C}\left|\varepsilon^{1-2 \kappa} M_{v / \varepsilon}\right| \geq \eta\right) \leq 2 \exp \left(-\frac{\eta^{2}}{2\left(\ln \varepsilon^{1-2 \kappa}+l C \varepsilon^{1-4 \kappa}\right)}\right)
$$

Consequently for $\kappa<\frac{1}{6}$,

$$
\varepsilon^{2 \kappa} \log \mathrm{P}\left(\sup _{0 \leq v \leq C}\left|\varepsilon^{1-2 \kappa} M_{v / \varepsilon}\right| \geq \eta\right) \leq \varepsilon^{2 \kappa} \log 2-\frac{\eta^{2}}{\varepsilon^{1-6 \kappa} 2 l\left(\eta \varepsilon^{2}+C\right)}
$$

and (5.7) follows.

So, it is left to verify (5.2) for $Z_{t}^{\varepsilon}:=\varepsilon^{1-\kappa} \int_{0}^{t} \int_{0}^{X_{v}^{\varepsilon} / \varepsilon} \theta(s) d s \sigma\left(X_{v}^{\varepsilon} / \varepsilon\right) d B_{v}$. Since $Z_{t}^{\varepsilon}$ is a continuous martingale with $\left\langle Z^{\varepsilon}\right\rangle_{t}=\varepsilon^{2(1-\kappa)} \int_{0}^{t}\left(\int_{0}^{X_{v}^{\varepsilon} / \varepsilon} \theta(s) d s\right)^{2} \sigma^{2}\left(X_{v}^{\varepsilon} / \varepsilon\right) d v$ and $\sigma^{2} \leq l$ we have

$$
\left\langle Z^{\varepsilon}\right\rangle_{T} \leq T l \sup _{|v| \leq C}\left(\varepsilon^{1-\kappa} \int_{0}^{v / \varepsilon} \theta(s) d s\right)^{2}, \quad \text { on the set } \Omega \backslash \Upsilon_{C}^{\varepsilon} .
$$


on the set $\Omega \backslash \Upsilon_{C}^{\varepsilon}$, For $r>0$, write

$$
\begin{aligned}
& \mathrm{P}\left(\sup _{t \leq T}\left|Z_{t}^{\varepsilon}\right|>\eta, \Omega \backslash \Upsilon_{C}^{\varepsilon}\right) \leq \mathrm{P}\left(\sup _{t \leq T}\left|Z_{t}^{\varepsilon}\right|>\eta,\left\langle Z^{\varepsilon}\right\rangle_{T} \leq T l \sup _{|v| \leq C}\left|\varepsilon^{1-\kappa} \int_{0}^{v / \varepsilon} \theta(s) d s\right|^{2}\right) \\
& \leq 2\left\{\mathrm{P}\left(\sup _{t \leq T}\left|Z_{t}^{\varepsilon}\right|>\eta,\left\langle Z^{\varepsilon}\right\rangle_{T} \leq 2 l r^{2} \varepsilon^{2 \kappa}\right) \bigvee 2 \mathrm{P}\left(\sup _{0 \leq v \leq C}\left|\varepsilon^{1-\kappa} \int_{0}^{v / \varepsilon} \theta(s) d s\right|>r \varepsilon^{\kappa}\right)\right\} \\
& \leq 4 \exp \left(\frac{-\eta^{2}}{4 l r^{2} \varepsilon^{2 \kappa}}\right) \bigvee 4 \mathrm{P}\left(\sup _{0 \leq v \leq C}\left|\varepsilon^{1-2 \kappa} \int_{0}^{v / \varepsilon} \theta(s) d s\right|>r\right) .
\end{aligned}
$$

Since $r$ in (5.8) is an arbitrary positive parameter, we have

$$
\begin{aligned}
& \varlimsup_{\varepsilon \rightarrow 0} \varepsilon^{2 \kappa} \log \mathrm{P}\left(\sup _{t \leq T}\left|Z_{t}^{\varepsilon}\right|>\eta, \Omega \backslash \Upsilon_{C}^{\varepsilon}\right) \\
& \leq \varepsilon^{2 \kappa} \log 4-\frac{\eta^{2}}{4 l r^{2}} \bigvee \varlimsup_{\varepsilon \rightarrow 0} \varepsilon^{2 \kappa} \log \mathrm{P}\left(\sup _{0<v \leq C}\left|\varepsilon^{1-2 \kappa} \int_{0}^{v / \varepsilon} \theta(s) d s\right|>r\right)=-\frac{\eta^{2}}{4 l r^{2}} \underset{r \rightarrow 0}{\longrightarrow}-\infty,
\end{aligned}
$$

where the equality is due to (5.6) proved above.

The proof for the second part in (1.4) is similar: we introduce the stationary process

$$
\theta(t)=1-\frac{\mathbf{a}}{\sigma^{2}(t)}
$$

where $\mathbf{a}$ is a fixed constant such that $\mathrm{E} \theta(t)=0$, i.e. $\mathbf{a}=1 / \sum_{i=1}^{m} \frac{\pi_{i}}{a_{i}^{2}}$, and set $H(x)=$ $\int_{0}^{x} \int_{0}^{v} \theta(s) d s d v$. By Krylov-Itô's formula [7],

$$
H\left(X_{t}^{\varepsilon} / \varepsilon\right)=H\left(x_{0} / \varepsilon\right)+\frac{1}{\varepsilon} \int_{0}^{t} \int_{0}^{X_{v}^{\varepsilon} / \varepsilon} \theta(s) d s d X_{v}^{\varepsilon}+\frac{1}{\varepsilon^{2(1-\kappa)}} \int_{0}^{t}\left[\sigma^{2}\left(X_{s}^{\varepsilon} / \varepsilon\right)-\mathbf{a}\right] d s
$$

or, equivalently,

$$
\begin{aligned}
& \int_{0}^{t}\left[\sigma^{2}\left(X_{s}^{\varepsilon} / \varepsilon\right)-\mathbf{a}\right] d s=\varepsilon^{2(1-\kappa)} \int_{0}^{X_{t}^{\varepsilon} / \varepsilon} \int_{0}^{v} \theta(s) d s d v-\varepsilon^{2(1-\kappa)} \int_{0}^{x_{0} / \varepsilon} \int_{0}^{v} \theta(s) d s d v- \\
& \varepsilon^{1-2 \kappa} \int_{0}^{t} \int_{0}^{X_{v}^{\varepsilon} / \varepsilon} \theta(s) d s b\left(X_{v}^{\varepsilon} / \varepsilon\right) d v-\varepsilon^{1-\kappa} \int_{0}^{t} \int_{0}^{X_{v}^{\varepsilon} / \varepsilon} \theta(s) d s \sigma\left(X_{v}^{\varepsilon} / \varepsilon\right) d B_{v} .
\end{aligned}
$$

Other steps of the proof repeat the previous ones and are omitted.

\section{MDP GAP BETWEEN OSCILLATING AND RANDOM ENVIRONMENTS}

The MDP regimes for oscillating and random environments are proved for different ranges of $\kappa$ :

$$
\kappa \in\left(0, \frac{1}{2}\right) \quad \text { and } \quad \kappa \in\left(0, \frac{1}{6}\right)
$$

respectively. This fact is explained by faster homogenization effect of the oscillating environment than the random one. This is clearly seen from the following proof sketch of the convergence in (1.4) for the oscillating environment. 
Denote by $\theta(s)$ either $\frac{b(s)-\mathbf{b}}{\sigma^{2}(s)}$ or $1-\frac{\mathbf{a}}{\sigma^{2}(s)}$ and choose $\mathbf{b}$ and $\mathbf{a}$ to satisfy the condition

$$
\int_{0}^{1} \theta(s) d s=0
$$

that is, $\mathbf{b}=\int_{0}^{1} \frac{b(s) d s}{\sigma^{2}(s)} / \int_{0}^{1} \frac{d s}{\sigma^{2}(s)} \quad$ and $\quad \mathbf{a}=1 / \int_{0}^{1} \frac{1}{\sigma^{2}(s)} d s$. Set $H(x)=\int_{0}^{x} \int_{0}^{v} \theta(s) d s d v$. Applying the Itô formula to $\varepsilon^{2(1-\kappa)} H\left(X_{t}^{\varepsilon} / \varepsilon\right)$, we find that

$$
\begin{aligned}
& \left.\begin{array}{l}
\int_{0}^{t}\left[b\left(X_{s}^{\varepsilon} / \varepsilon\right)-\mathbf{b}\right] d s \\
\int_{0}^{t}\left[\sigma^{2}\left(X_{s}^{\varepsilon} / \varepsilon\right)-\mathbf{a}\right] d s
\end{array}\right\}=\varepsilon^{2(1-\kappa)} \int_{0}^{X_{t}^{\varepsilon} / \varepsilon} \int_{0}^{v} \theta(s) d s d v \\
& -\varepsilon^{2(1-\kappa)} \int_{0}^{x_{0} / \varepsilon} \int_{0}^{v} \theta(s) d s d v \\
& -\varepsilon^{1-2 \kappa} \int_{0}^{t} \int_{0}^{X_{v}^{\varepsilon} / \varepsilon} \theta(s) d s b\left(X_{v}^{\varepsilon} / \varepsilon\right) d v \\
& -\varepsilon^{1-\kappa} \int_{0}^{t} \int_{0}^{X_{v}^{\varepsilon} / \varepsilon} \theta(s) d s \sigma\left(X_{v}^{\varepsilon} / \varepsilon\right) d B_{v} .
\end{aligned}
$$

Since $\theta(s)$ is a periodic function, (6.1) implies that $\left|\int_{0}^{t} \theta(s) d s\right|$ is bounded uniformly in $t$. This is the origin of strong homogenization. Namely, we have the following estimates for the terms in (6.2) - 6.4) (here $l$ is a generic positive constant):

$$
\begin{aligned}
& \varepsilon^{2(1-\kappa)}\left|\int_{0}^{X_{t}^{\varepsilon} / \varepsilon} \int_{0}^{v} \theta(s) d s d v\right| \leq l \varepsilon^{1-2 \kappa} \sup _{t \leq T}\left|X_{t}^{\varepsilon}\right| \\
& \varepsilon^{2(1-\kappa)}\left|\int_{0}^{x_{0} / \varepsilon} \int_{0}^{v} \theta(s) d s d v\right| \leq l \varepsilon^{1-2 \kappa}\left|x_{0}\right| \\
& \varepsilon^{1-2 \kappa}\left|\int_{0}^{t} \int_{0}^{X_{v}^{\varepsilon} / \varepsilon} \theta(s) d s b\left(X_{v}^{\varepsilon} / \varepsilon\right) d v\right| \leq l \varepsilon^{1-2 \kappa} .
\end{aligned}
$$

The second and third upper bounds are deterministic and so the corresponding terms are exponentially tight with the rate $\varepsilon^{2 \kappa}$ for any $\kappa>0$. Since $b$ and $\sigma$ are bounded, using (4.4), we have

$$
\lim _{C \rightarrow \infty} \varlimsup_{\varepsilon \rightarrow 0} \varepsilon^{2 \kappa} \log \mathrm{P}\left(\sup _{t \leq T}\left|X_{t}^{\varepsilon}\right|>C\right)=-\infty
$$

and, hence, the first term is exponentially tight for any $\kappa>0$ as well.

The restriction for $\kappa$ is imposed by the exponential negligibility with the rate $\varepsilon^{2 \kappa}$ of the continuous martingale $Z_{t}^{\varepsilon}:=\varepsilon^{1-\kappa} \sup _{t \leq T}\left|\int_{0}^{t} \int_{0}^{X_{v}^{\varepsilon} / \varepsilon} \theta(s) d s \sigma\left(X_{v}^{\varepsilon} / \varepsilon\right) d B_{v}\right|$. Since

$$
\left\langle Z^{\varepsilon}\right\rangle_{t}=\varepsilon^{2(1-\kappa)} \int_{0}^{t}\left(\int_{0}^{X_{v}^{\varepsilon} / \varepsilon} \theta(s) d s\right)^{2} \sigma^{2}\left(X_{v}^{\varepsilon} / \varepsilon\right) d v
$$

and $\left|\int_{0}^{t} \theta(s) d s\right|$ is uniformly bounded in $t(!)$,

$$
\left\langle Z^{\varepsilon}\right\rangle_{T} \leq \varepsilon^{2(1-\kappa)} l .
$$


Therefore, by Lemma 4.1 .

$$
\mathrm{P}\left(\sup _{t \leq T}\left|Z_{t}^{\varepsilon}\right|>\eta\right)=\mathrm{P}\left(\sup _{t \leq T}\left|Z_{t}^{\varepsilon}\right|>\eta,\left\langle Z^{\varepsilon}\right\rangle_{T} \leq \varepsilon^{2(1-\kappa)} l\right) \leq 2 \exp \left(-\frac{\eta^{2}}{2 l \varepsilon^{2(1-\kappa)}}\right),
$$

and so, $\varepsilon^{2 \kappa} \log \mathrm{P}\left(\sup _{t \leq T}\left|Z_{t}^{\varepsilon}\right|>\eta\right) \leq \varepsilon^{2 \kappa} \log 2-\frac{\eta^{2}}{2 l \varepsilon^{2(1-2 \kappa)}} \underset{\varepsilon \rightarrow 0}{\longrightarrow}-\infty$ only if $\kappa<\frac{1}{2}$.

\section{Appendix A. The LDP ANALYSIS}

The use of (1.4) makes the proof of Theorem 3.1 transparent. As was mentioned, the implication

$$
\text { (1.4) } \Rightarrow \text { the statement of Theorem } 3.1
$$

follows from Corollary 4.3.8 in [13] (see also Corollary 6.7 in [14]) which are applicable not only to the setting under consideration but also to various classes of semimartingales. For reader's convenience, we show how (A.1) works in our setting.

Let $X^{\varepsilon}=\left(X_{t}^{\varepsilon}\right)_{t \leq T}$ be a continuous semimartingale defined on a stochastic basis, with the general conditions, $\left(\Omega, \mathbf{F}, \mathcal{F}^{\varepsilon}=\left(\mathscr{F}_{t}^{\varepsilon}\right)_{t \leq T}, \mathrm{P}\right)$ :

$$
X_{t}^{\varepsilon}=x_{0}+\int_{0}^{t} b_{s}^{\varepsilon} d s+\varepsilon^{\kappa} \int_{0}^{t} \alpha_{s}^{\varepsilon} d B_{s},
$$

where the Brownian motion $B_{t}$ and the processes $b_{t}^{\varepsilon}, \alpha_{t}^{\varepsilon}$ are $\mathcal{F}^{\varepsilon}$-adapted (with $\int_{0}^{T}\left|b_{t}^{\varepsilon}\right| d t<\infty$, $\left.\int_{0}^{T}\left(\alpha_{s}^{\varepsilon}\right)^{2} d t<\infty\right)$, a.s. $\varepsilon$ is a small positive parameter, $\kappa$ is a positive number.

Theorem A.1. Assume $0<c_{1} \leq\left|\alpha_{s}^{\varepsilon}\right|^{2} \leq c_{2}$ and $\left|\beta_{s}^{\varepsilon}\right| \leq c_{3}$ and there exist constants $\mathbf{b}$ and $\mathbf{a}>0$ such that for any $\eta>0$

$$
\begin{aligned}
& \lim _{\varepsilon \rightarrow 0} \varepsilon^{2 \kappa} \log \mathrm{P}\left(\sup _{t \leq T}\left|\int_{0}^{T}\left[b_{s}^{\varepsilon}-\mathbf{b}\right] d s\right|>\eta\right)=-\infty \\
& \lim _{\varepsilon \rightarrow 0} \varepsilon^{2 \kappa} \log \mathrm{P}\left(\sup _{t \leq T}\left|\int_{0}^{T}\left[\left(\alpha_{s}^{\varepsilon}\right)^{2}-\mathbf{a}\right] d s\right|>\eta\right)=-\infty .
\end{aligned}
$$

Then, the family $\left\{X^{\varepsilon}\right\}_{\varepsilon \rightarrow 0}$ obeys the LDP with the rate $\varepsilon^{2 \kappa}$ and the rate function

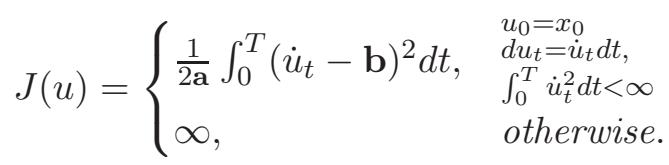

The proof of this theorem uses a standard fact (see, e.g. [12]):

$$
\left.\begin{array}{l}
\text { Exponential Tightness } \\
\text { Local LDP }
\end{array}\right\} \Rightarrow \text { LDP. }
$$

For the proof of exponential tightness and local LDP it is convenient to use the stopping time

$$
\tau_{\varepsilon, \zeta}=\inf \left\{t \leq T:\left|\int_{0}^{t}\left[b_{s}^{\varepsilon}-\mathbf{b}\right] d s\right|+\left|\int_{0}^{t}\left[\left(\alpha_{s}^{\varepsilon}\right)^{2}-\mathbf{a}\right] d s\right|>\zeta\right\}
$$


Notice also that (A.2) and (A.3) imply $\lim _{\varepsilon \rightarrow 0} \varepsilon^{2 \kappa} \log \mathrm{P}\left(\tau_{\varepsilon, \zeta}<\infty\right)=-\infty$ and, therefore, for any measurable set $\mathfrak{B}$,

$$
\varlimsup_{\varepsilon \rightarrow 0} \varepsilon^{2 \kappa} \log \mathrm{P}(\mathfrak{B}) \leq \varlimsup_{\zeta \rightarrow 0} \varlimsup_{\varepsilon \rightarrow 0} \varepsilon^{2 \kappa} \log \mathrm{P}\left(\mathfrak{B} \cap\left\{\tau_{\varepsilon, \zeta}=\infty\right\}\right) .
$$

A.1. Exponential tightness. Following [12], we shall prove that

$$
\begin{aligned}
& \lim _{C \rightarrow \infty} \varlimsup_{\varepsilon \rightarrow 0} \varepsilon^{2 \kappa} \log \mathrm{P}\left(\sup _{t \leq T}\left|X_{t}^{\varepsilon}\right|>C\right)=-\infty \\
& \lim _{\delta \rightarrow 0} \varlimsup_{\varepsilon \rightarrow 0} \varepsilon^{2 \kappa} \log \sup _{\theta<T} \mathrm{P}\left(\sup _{0<t \leq \delta}\left|X_{\theta+t}^{\varepsilon}-X_{\theta}^{\varepsilon}\right|>\eta\right)=-\infty, \forall \eta>0,
\end{aligned}
$$

where $\theta$ is $\mathcal{F}^{\varepsilon}$-stopping time.

A.1.1. The proof of (A.5). Set $\mathfrak{B}:=\left\{\sup _{t \leq T}\left|X_{t}^{\varepsilon}\right|>C\right\}$. Due to (A.4), it suffices to prove that $\lim _{C \rightarrow \infty} \varlimsup_{\lim _{\varepsilon \rightarrow 0}} \varepsilon^{2 \kappa} \log \mathrm{P}\left(\sup _{t \leq T}\left|X_{t}^{\varepsilon}\right|>C, \tau_{\varepsilon, \zeta}=\infty\right)=-\infty$. To this end, using the random variable $\left|x_{0}\right|+|\mathbf{b}| T+\zeta+\sup _{t \leq T}\left|\varepsilon^{\kappa} \int_{0}^{t \wedge \tau_{\varepsilon, \zeta}} \alpha_{s}^{\varepsilon} d B_{s}\right|$ as an upper bound for $\sup _{t \leq T}\left|X_{t}^{\varepsilon}\right|$ on the set $\left\{\tau_{\varepsilon, \zeta}=\infty\right\}$, the proof reduces to

$$
\lim _{C \rightarrow \infty} \varlimsup_{\varepsilon \rightarrow 0} \varepsilon^{2 \kappa} \log \mathrm{P}\left(\sup _{t \leq T}\left|\varepsilon^{\kappa} \int_{0}^{t \wedge \tau_{\varepsilon, \zeta}} \alpha_{s}^{\varepsilon} d B_{s}\right|>C, \tau_{\varepsilon, \zeta}=\infty\right)=-\infty .
$$

Applying (4.4) to $M_{t}=\varepsilon^{\kappa} \int_{0}^{t \wedge \tau_{\varepsilon, \zeta}} \alpha_{s}^{\varepsilon} d B_{s}$ and taking into account that

$$
\langle M\rangle_{T}=\varepsilon^{2 \kappa} \int_{0}^{T \wedge \tau_{\varepsilon, \zeta}}\left(\alpha_{s}^{\varepsilon}\right)^{2} d s \leq \varepsilon^{2 \kappa}[\mathbf{a} T+\zeta]
$$

we find the following upper bound $\mathrm{P}\left(\sup _{t \leq T}\left|M_{t}\right|>C\right) \leq 2 \exp \left(-\frac{C^{2}}{2 \varepsilon^{2 \kappa}(\mathbf{a} T+\zeta)}\right)$ which, in turn, provides (A.5).

A.1.2. The proof of (A.6). Let $M_{t}$ be the same as above and let $\mathcal{F}^{\varepsilon, \theta}=\left(\mathscr{F}_{\theta+t}^{\varepsilon}\right)_{t>0}$. Denote $N_{t}^{\theta}:=\left(M_{\theta+t}-M_{\theta}\right)$. The random process $N_{t}^{\theta}$ is a martingale relative to $\mathcal{F}^{\varepsilon, \theta}$ with $\left\langle N^{\theta}\right\rangle_{t}=\varepsilon^{2 \kappa} \int_{\theta}^{\theta+t}\left(\alpha_{s}^{\varepsilon}\right)^{2} d s$. Denote $L_{t}^{\varepsilon}:=\int_{\theta}^{\theta+t} b_{s}^{\varepsilon} d s+N_{t}^{\theta}$ and notice that (A.6) is nothing but

$$
\lim _{\zeta \rightarrow 0} \varlimsup_{\delta \rightarrow 0} \varlimsup_{\varepsilon \rightarrow 0} \varepsilon^{2 \kappa} \log \sup _{\theta<T} \mathrm{P}\left(\sup _{0<t \leq \delta}\left|L_{t}^{\varepsilon}\right|>\eta\right)=-\infty,
$$

so that, in view of (A.4), it suffices to prove that

$$
\lim _{\zeta \rightarrow 0} \varlimsup_{\delta \rightarrow 0} \varlimsup_{\varepsilon \rightarrow 0} \varepsilon^{2 \kappa} \log \sup _{\theta<T} \mathrm{P}\left(\sup _{0<t \leq \delta}\left|L_{t}^{\varepsilon}\right|>\eta, \tau_{\varepsilon, \zeta}=\infty\right)=-\infty .
$$

or, to verify the stronger condition

$$
\lim _{\zeta \rightarrow 0} \varlimsup_{\delta \rightarrow 0} \varlimsup_{\varepsilon \rightarrow 0} \varepsilon^{2 \kappa} \log \sup _{\theta<T} \mathrm{P}\left(\sup _{0<t \leq \delta \wedge \tau_{\varepsilon, \zeta}}\left|L_{t}^{\varepsilon}\right|>\eta\right)=-\infty .
$$

Obviously, $\sup _{0<t \leq \delta}\left|L_{t}^{\varepsilon}\right| \leq \delta|\mathbf{b}|+\zeta+\sup _{0<t \leq \delta}\left|N_{t \wedge \tau_{\varepsilon, \zeta}}^{\theta}\right|$. For fixed $\eta$ we choose sufficiently small $\delta$ and $\zeta$ such that $\delta|\mathbf{b}|+\zeta<\eta$. Now, instead of (A.7) we have to prove

$$
\lim _{\zeta \rightarrow 0} \varlimsup_{\delta \rightarrow 0} \varlimsup_{\varepsilon \rightarrow 0} \varepsilon^{2 \kappa} \log \sup _{\theta<T} \mathrm{P}\left(\sup _{0<t \leq \delta \wedge \tau_{\varepsilon, \zeta}}\left|N_{t}^{\theta}\right|>\eta-\delta|\mathbf{b}|-\zeta\right)=-\infty .
$$


Further, due to $\left\langle N^{\theta}\right\rangle_{\delta \wedge \tau_{\varepsilon, \zeta}}=\varepsilon^{2 \kappa} \int_{\theta \wedge \tau_{\varepsilon, \zeta}}^{\left(\theta+\delta \wedge \tau_{\varepsilon, \zeta}\right)}\left(\alpha_{s}^{\varepsilon}\right)^{2} d s \leq \varepsilon^{2 \kappa}[\delta \mathbf{a}+\zeta]$, by applying (4.4) to $N_{t \wedge \tau_{\varepsilon, \zeta}}=\varepsilon^{\kappa} \int_{0}^{t \wedge \tau_{\varepsilon, \zeta}} \alpha_{s}^{\varepsilon} d B_{s}$ we find the following upper bound

$$
\mathrm{P}\left(\sup _{t \leq \delta \wedge \tau_{\varepsilon, \zeta}}\left|N_{t}^{\theta}\right|>\eta-\delta|\mathbf{b}|-\zeta\right) \leq 2 \exp \left(-\frac{(\eta-\delta|\mathbf{b}|-\zeta)^{2}}{2 \varepsilon^{2 \kappa}(\delta \mathbf{a}+\zeta)}\right)
$$

which gives (A.6).

A.2. Local LDP. It is well known that for exponentially tight family $\left\{\left(X_{t}^{\varepsilon}\right)_{t \leq T}\right\}_{\varepsilon \rightarrow 0}$ the rate function coincides with the local rate function $J(u)$ determined by the conditions: for any $u \in \mathbb{C}_{[0, T]}$,

$$
\begin{aligned}
& \varlimsup_{\delta \rightarrow 0} \varlimsup_{\varepsilon \rightarrow 0} \varepsilon^{2 \kappa} \log \mathrm{P}\left(\sup _{t \leq T}\left|X_{t}^{\varepsilon}-u_{t}\right| \leq \delta\right) \leq-J(u) \\
& \varliminf_{\delta \rightarrow 0} \varlimsup_{\varepsilon \rightarrow 0} \varepsilon^{2 \kappa} \log \mathrm{P}\left(\sup _{t \leq T}\left|X_{t}^{\varepsilon}-u_{t}\right| \leq \delta\right) \geq-J(u) .
\end{aligned}
$$

Since the range space of $J(u)$ is the interval $[0, \infty]$, we compute separately $J(u)$ on two sets: $\mathfrak{U}_{1}=\left\{u \in \mathbb{C}_{[0, T]}: J(u)<\infty\right\}$ and $\mathfrak{U}_{2}=\left\{u \in \mathbb{C}_{[0, T]}: J(u)=\infty\right\}$.

\section{A.2.1. Upper bound under $J(u)<\infty$.}

Lemma A.1. (A.8) holds with $J(u)=\frac{1}{2 \mathbf{a}} \int_{0}^{T}\left(\dot{u}_{t}-\mathbf{b}\right)^{2} d t$ for any

$$
u \in\left\{u_{0}=x_{0}, d u_{t}=\dot{u}_{t} d t, \int_{0}^{T} \dot{u}_{t}^{2} d t<\infty\right\}=: \mathfrak{U}_{1} .
$$

Proof. Denote $\mathfrak{B}_{\delta}=\left\{\sup _{t \leq T}\left|X_{t}^{\varepsilon}-u_{t}\right| \leq \delta\right\}$. In view of (A.4), it suffices to show that

$$
\varlimsup_{\zeta \rightarrow 0} \varlimsup_{\delta \rightarrow 0} \varlimsup_{\varepsilon \rightarrow 0} \varepsilon^{2 \kappa} \log \mathrm{P}\left(\mathfrak{B}_{\delta} \cap\left\{\tau_{\varepsilon, \zeta}=\infty\right\}\right) \leq-\frac{1}{2 \mathbf{a}} \int_{0}^{T}\left(\dot{u}_{t}-\mathbf{b}\right)^{2} d t
$$

To this end, we introduce a martingale exponential

$$
\mathfrak{z} t=\exp \left(\frac{1}{\varepsilon^{2 \kappa}}\left[\int_{0}^{T} \lambda(s)\left[d X_{s}^{\varepsilon}-b_{s}^{\varepsilon} d s\right]-\frac{1}{2} \int_{0}^{T} \lambda^{2}(s)\left(a_{s}^{\varepsilon}\right)^{2} d s\right]\right), t \leq T,
$$

where $\lambda(s)$ is a continuously differentiable function with the derivative $\dot{\lambda}(s)$. Integrating by parts with the help of Itô's formula we find that $\int_{0}^{T} \lambda(s) d X_{s}^{\varepsilon}=\lambda(T) X_{T}^{\varepsilon}-\lambda(0) x_{0}-$ $\int_{0}^{T} X_{s}^{\varepsilon} \dot{\lambda}(s) d s$ and rewrite $\log \mathfrak{z} t$ to the following form:

$$
\log \mathfrak{z}_{T}=\frac{1}{\varepsilon^{2 \kappa}}\left[\lambda(T) X_{T}^{\varepsilon}-\lambda(0) x_{0}-\int_{0}^{T} X_{s}^{\varepsilon} \dot{\lambda}(s) d s-\int_{0}^{T}\left(\lambda(s) b_{s}^{\varepsilon}+\frac{\lambda^{2}(s)}{2}\left(a_{s}^{\varepsilon}\right)^{2}\right) d s\right] .
$$


Write

$$
\begin{aligned}
& \lambda(T) X_{T}^{\varepsilon}-\lambda(0) x_{0}-\int_{0}^{T} X_{s}^{\varepsilon} \dot{\lambda}(s) d s-\int_{0}^{T}\left(\lambda(s) b_{s}^{\varepsilon}+\frac{\lambda^{2}(s)}{2}\left(a_{s}^{\varepsilon}\right)^{2}\right) d s \\
& =\lambda(T) u_{T}-\lambda(0) u_{0}-\int_{0}^{T} u_{s} \dot{\lambda}(s) d s-\int_{0}^{T}\left(\lambda(s) \mathbf{b}+\frac{\lambda^{2}(s)}{2} \mathbf{a}\right) d s \\
& \quad+\lambda(T)\left[X_{t}^{\varepsilon}-u_{T}\right]-\int_{0}^{T}\left[X_{s}^{\varepsilon}-u_{s}\right] \dot{\lambda}(s) d s-\int_{0}^{T}\left(\lambda(s)\left[b_{s}^{\varepsilon}-\mathbf{b}\right]+\frac{\lambda^{2}(s)}{2}\left[\left(\alpha_{s}^{\varepsilon}\right)^{2}-\mathbf{a}\right]\right) d s .
\end{aligned}
$$

Integrating by parts we find that

$$
\begin{aligned}
& \int_{0}^{T} \lambda(s)\left[b_{s}^{\varepsilon}-\mathbf{b}\right] d s=\lambda(T) \int_{0}^{T}\left[b_{s}^{\varepsilon}-\mathbf{b}\right] d s-\int_{0}^{T} \dot{\lambda}(s) \int_{0}^{s}\left[b_{s^{\prime}}^{\varepsilon}-\mathbf{b}\right] d s^{\prime} d s \\
& \int_{0}^{T} \frac{\lambda^{2}(s)}{2}\left[\left(a_{s}^{\varepsilon}\right)^{2}-\mathbf{a}\right] d s=\frac{\lambda^{2}(T)}{2} \int_{0}^{T}\left[\left(a_{s}^{\varepsilon}\right)^{2}-\mathbf{a}\right] d s-\int_{0}^{T} \lambda(s) \dot{\lambda}(s) \int_{0}^{s}\left[\left(a_{s^{\prime}}^{\varepsilon}\right)^{2}-\mathbf{a}\right] d s^{\prime} d s,
\end{aligned}
$$

and transform (A.10 into

$$
\begin{aligned}
\lambda(T) X_{T}^{\varepsilon}-\lambda(0) x_{0}-\int_{0}^{T} X_{s}^{\varepsilon} \dot{\lambda}(s) d s-\int_{0}^{T}\left(\lambda(s) b_{s}^{\varepsilon}+\frac{\lambda^{2}(s)}{2}\left(a_{s}^{\varepsilon}\right)^{2}\right) d s \\
=\lambda(T) u_{T}-\lambda(0) u_{0}-\int_{0}^{T} u_{s} \dot{\lambda}(s) d s-\int_{0}^{T}\left(\lambda(s) \mathbf{b}+\frac{\lambda^{2}(s)}{2} \mathbf{a}\right) d s \\
\quad+\lambda(T)\left[X_{t}^{\varepsilon}-u_{T}\right]-\int_{0}^{T}\left[X_{s}^{\varepsilon}-u_{s}\right] \dot{\lambda}(s) d s \\
\quad-\lambda(T) \int_{0}^{T}\left[b_{s}^{\varepsilon}-\mathbf{b}\right] d s+\int_{0}^{T} \dot{\lambda}(s) \int_{0}^{s}\left[b_{s^{\prime}}^{\varepsilon}-\mathbf{b}\right] d s^{\prime} d s \\
\quad-\frac{\lambda^{2}(T)}{2} \int_{0}^{T}\left[\left(a_{s}^{\varepsilon}\right)^{2}-\mathbf{a}\right] d s+\int_{0}^{T} \lambda(s) \dot{\lambda}(s) \int_{0}^{s}\left[\left(a_{s^{\prime}}^{\varepsilon}\right)^{2}-\mathbf{a}\right] d s^{\prime} d s .
\end{aligned}
$$

The right hand side of this identity can be estimated from below on the set $\mathfrak{B}_{\delta} \cap\left\{\tau_{\varepsilon, \zeta}=\infty\right\}$ by

$$
\begin{aligned}
\lambda(T) & u_{T}-\lambda(0) u_{0}-\int_{0}^{T} u_{s} \dot{\lambda}(s) d s-\int_{0}^{T}\left(\lambda(s) \mathbf{b}+\frac{\lambda^{2}(s)}{2} \mathbf{a}\right) d s \\
& -\delta\left(|\lambda(T)|+\int_{0}^{T}|\dot{\lambda}(s)| d s\right) \\
& -\zeta\left(|\lambda(T)|+\int_{0}^{T}|\dot{\lambda}(s)| d s+\frac{\lambda^{2}(T)}{2}+\int_{0}^{T}|\lambda(s) \dot{\lambda}(s)| d s\right) .
\end{aligned}
$$

With $l_{1}=|\lambda(T)|+\int_{0}^{T}|\dot{\lambda}(s)| d s+|\lambda(T)|+\int_{0}^{T}|\dot{\lambda}(s)| d s+\frac{\lambda^{2}(T)}{2}+\int_{0}^{T}|\lambda(s) \dot{\lambda}(s)| d s$ and the identity $\lambda(T) u_{T}-\lambda(0) u_{0}-\int_{0}^{T} u_{s} \dot{\lambda}(s) d s=\int_{0}^{T} \lambda(s) \dot{u}_{t} d t$ we find the following lower bound for $\mathfrak{z} T$ :

$$
\mathfrak{z}_{*}=\exp \left(\frac{1}{\varepsilon^{2 \kappa}}\left[\int_{0}^{T}\left[\lambda(s)\left(\dot{u}_{s}-\mathbf{b}\right)-\frac{\lambda^{2}(s)}{2}\right] d s-l_{1}(\delta+\zeta)\right]\right)
$$


The martingale exponential $\mathfrak{z} t$ is a positive local martingale and a supermartingale too. Hence, $\mathrm{E}_{\mathfrak{z} T} \leq 1$. This bound implies $\mathrm{E}_{\left\{\mathfrak{B}_{\delta} \cap\left(\tau_{\varepsilon, \zeta}=\infty\right)\right\}} \mathfrak{z} T \leq 1$ and, in turn,

$$
\mathrm{E} I_{\left\{\mathfrak{B}_{\delta} \cap\left(\tau_{\varepsilon, \zeta}=\infty\right)\right\} \mathfrak{z} *} \leq 1 .
$$

Jointly with (A.11) the latter implies

$$
\varlimsup_{\zeta \rightarrow 0} \varlimsup_{\delta \rightarrow 0} \varlimsup_{\varepsilon \rightarrow 0} \varepsilon^{2 \kappa} \log \mathrm{P}\left(\mathfrak{B}_{\delta} \cap\left\{\tau_{\varepsilon, \zeta}=\infty\right\}\right) \leq-\int_{0}^{T}\left\{\lambda(s)\left[\dot{u}_{s}-\mathbf{b}\right]-\frac{\lambda^{2}(s)}{2} \mathbf{a}\right\} d s .
$$

Recall that A.12) is valid provided that $\lambda(s)$ is a continuously differentiable function. Assume that $\dot{u}_{s}$ is also continuously differentiable. Then taking $\lambda(s) \equiv \frac{\dot{u}_{s}-\mathbf{b}}{\mathbf{a}}$ we obtain the desired result. In the general case, $\dot{u}_{t}$ is only a density of $u_{t}$ relative to $d t$, so that, $\lambda(t)$ as chosen above may not be continuously differentiable. In this case we use the identity

$$
-\int_{0}^{T}\left\{\lambda_{m}(s)\left[\dot{u}_{s}-\mathbf{b}\right]-\frac{\lambda_{m}^{2}(s)}{2} \mathbf{a}\right\} d s=-\int_{0}^{T} \frac{\left(\dot{u}_{s}-\mathbf{b}\right)^{2}}{2 \mathbf{a}} d s+\int_{0}^{T} \frac{\mathbf{a}}{2}\left(\lambda_{m}(s)-\frac{\dot{u}_{s}-\mathbf{b}}{\mathbf{a}}\right)^{2} d s
$$

where $\lambda_{m}(s)$ is a sequence of continuously differentiable functions such that

$$
\lim _{m \rightarrow \infty} \int_{0}^{T} \frac{\mathbf{a}}{2}\left(\lambda_{m}(s)-\frac{\dot{u}_{s}-\mathbf{b}}{\mathbf{a}}\right)^{2} d s=0
$$

A.2.2. Upper bound under $\boldsymbol{J}(\boldsymbol{u})=\infty$. Since $\mathfrak{U}_{2}=\mathbb{C}_{[0, T]} \backslash \mathfrak{U}_{1}$, it suffices to verify the upper bound in (A.8) under the following conditions:

(c.1) $u_{0}=x_{0}, d u_{t} \ll d t, \int_{0}^{T} \dot{u}^{2} d t=\infty$

(c.2) $u_{0}=x_{0}, d u_{t} \nless d t$

(c.3) $u_{0} \neq x_{0}$

Lemma A.2. For any of (c.1), (c.2) and (c.3), the upper bound in (A.8) holds with $J(u)=\infty$.

Proof.

(c.1) By (A.12) and (A.4), for any continuously differentiable function $\lambda(s)$, we have

$$
\varlimsup_{\delta \rightarrow 0} \varlimsup_{\varepsilon \rightarrow 0} \varepsilon^{2 \kappa} \log \mathrm{P}\left(\mathfrak{B}_{\delta}\right) \leq-\int_{0}^{T}\left\{\lambda(s)\left[\dot{u}_{s}-\mathbf{b}\right]-\frac{\lambda^{2}(s)}{2} \mathbf{a}\right\} d s .
$$

Let us take $\lambda_{n}(s)=\frac{\dot{u}_{s}-\mathbf{b}}{\mathbf{a}} I_{\left\{\left|\dot{u}_{s}\right| \leq n\right\}}$ and choose a sequence of continuously differentiable functions $\lambda_{m, n}(s)$ such that $\lim _{m \rightarrow \infty} \int_{0}^{T}\left[\lambda_{n}(s)-\lambda_{m, n}(s)\right]^{2}=0$. For $\lambda_{m, n}(s)$ A.13) implies:

$$
\varlimsup_{\delta \rightarrow 0} \varlimsup_{\varepsilon \rightarrow 0} \varepsilon^{2 \kappa} \log \mathrm{P}\left(\mathfrak{B}_{\delta}\right) \leq-\int_{0}^{T}\left\{\lambda_{m, n}(s)\left[\dot{u}_{s}-\mathbf{b}\right]-\frac{\lambda_{m, n}^{2}(s)}{2} \mathbf{a}\right\} d s .
$$

The right hand side of (A.14) converges to $-\int_{0}^{T}\left\{\lambda_{n}(s)\left[\dot{u}_{s}-\mathbf{b}\right]-\frac{\lambda_{n}^{2}(s)}{2} \mathbf{a}\right\} d s$ with $m \rightarrow \infty$. Noticing that $-\int_{0}^{T}\left\{\lambda_{n}(s)\left[\dot{u}_{s}-\mathbf{b}\right]-\frac{\lambda_{n}^{2}(s)}{2} \mathbf{a}\right\} d s=-\frac{1}{2 \mathbf{a}} \int_{0}^{T}\left(\dot{u}_{s}-\mathbf{b}\right)^{2} I_{\left\{\left|\dot{u}_{s}\right| \leq n\right\}} d s$, we find that for any $n \geq 1$,

$$
\varlimsup_{\delta \rightarrow 0} \varlimsup_{\varepsilon \rightarrow 0} \varepsilon^{2 \kappa} \log \mathrm{P}\left(\mathfrak{B}_{\delta}\right) \leq-\frac{1}{2 \mathbf{a}} \int_{0}^{T}\left(\dot{u}_{s}-\mathbf{b}\right)^{2} I_{\left\{\left|\dot{u}_{s}\right| \leq n\right\}} d s \underset{n \rightarrow \infty}{\longrightarrow}-\infty .
$$


(c.2) We show that $d u_{t} \nless d t$ enables us to choose a sequence $\left[s_{i}, t_{i}\right), i=1, \ldots n$ of nonoverlapping intervals on $[0, T]$ such that

$$
\lim _{n \rightarrow \infty} \sum_{i=1}^{n} \frac{\left|u_{t_{i}^{n}}-u_{s_{i}^{n}}\right|^{2}}{t_{i}^{n}-s_{i}^{n}}=\infty
$$

By the Cauchy-Schwarz inequality

$$
\left(\sum_{i=1}^{n}\left|u_{t_{i}^{n}}-u_{s_{i}^{n}}\right|\right)^{2} \leq \sum_{i=1}^{n}\left[t_{i}^{n}-s_{i}^{n}\right] \sum_{i=1}^{n} \frac{\left|u_{t_{i}^{n}}-u_{s_{i}^{n}}\right|^{2}}{t_{i}^{n}-s_{i}^{n}} .
$$

Further, for any small positive number $\gamma$ one can choose intervals $\left[s_{i}, t_{i}\right), i=1, \ldots n$ such that $\sum_{i=1}^{n}\left[t_{i}^{n}-s_{i}^{n}\right] \leq \gamma$ for any $n \geq n_{\gamma}$, where $n_{\gamma}$ is some number depending on $\gamma$ and, at the same time, $\sum_{i=1}^{n}\left|u_{t_{i}^{n}}-u_{s_{i}^{n}}\right| \geq D>0$. Hence, (A.16) implies $\frac{D^{2}}{\gamma} \leq \sum_{i=1}^{n} \frac{\left|u_{t_{i}^{n}}-u_{s_{i}^{n}}\right|^{2}}{t_{i}^{n}-s_{i}^{n}}$ and A.15) holds in view of $D$ remains strictly positive with $\gamma \rightarrow 0$.

We prove that

$$
\varlimsup_{\zeta \rightarrow 0} \varlimsup_{\delta \rightarrow 0} \varlimsup_{\varepsilon \rightarrow 0} \log \mathrm{P}\left(\mathfrak{B}_{\delta} \cap\left\{\tau_{\varepsilon, \zeta}=\infty\right\}\right) \leq-\frac{1}{2 \mathbf{a}}\left\{\mathbf{b}^{2} T-2 \mathbf{b}\left(u_{T}-u_{0}\right)+\sum_{i=1}^{n} \frac{\left[u_{t_{i}^{n}}-u_{s_{i}^{n}}\right]^{2}}{t_{i}^{n}-s_{i}^{n}}\right\}
$$

and, then, apply (A.15).

With $\lambda(t)=\sum_{i} \lambda_{i} I_{\left\{s_{i}^{n} \leq t<t_{i}^{n}\right\}}$, we introduce a martingale exponential

$$
\mathfrak{z}_{t}=\exp \left(\frac{1}{\varepsilon^{2 \kappa}}\left[\int_{0}^{T} \lambda(s)\left[d X_{s}^{\varepsilon}-b_{s}^{\varepsilon} d s\right]-\frac{1}{2} \int_{0}^{T} \lambda^{2}(s)\left(a_{s}^{\varepsilon}\right)^{2} d s\right]\right)
$$

and estimate it from below on the set $\mathfrak{B}_{\delta} \cap\left\{\tau_{\varepsilon, \zeta}=\infty\right\}$. Write

$$
\begin{aligned}
\log \mathfrak{z} T & =\frac{1}{\varepsilon^{2 \kappa}} \sum_{i=1}^{n}\left[\lambda_{i}\left[X_{t_{i}^{n}}^{\varepsilon}-X_{s_{i}^{n}}^{\varepsilon}\right]-\int_{s_{i}^{n}}^{t_{i}^{n}}\left\{\lambda_{i} b_{s}^{\varepsilon}+\frac{1}{2} \lambda_{i}^{2}\left(a_{s}^{\varepsilon}\right)^{2}\right\} d s\right] \\
\geq & \frac{1}{\varepsilon^{2 \kappa}} \sum_{i=1}^{n}\left\{\lambda_{i}\left[u_{t_{i}^{n}}-u_{s_{i}^{n}}\right]-\left[\lambda_{i} \mathbf{b}+\frac{\lambda_{i}^{2}}{2} \mathbf{a}\right]\left(t_{i}^{n}-s_{i}^{n}\right)\right\} \\
& -\frac{1}{\varepsilon^{2 \kappa}} \sum_{i=1}^{n}\left(2\left|\lambda_{i}\right| \delta+\zeta\left[\left|\lambda_{i}\right|+\frac{\lambda_{i}^{2}}{2}\right]\right)\left(t_{i}^{n}-s_{i}^{n}\right):=\log \mathfrak{z}_{*} .
\end{aligned}
$$

Since $\mathrm{E}_{\mathfrak{z} T} \leq 1$, also $\mathrm{E} I_{\left\{\mathfrak{B}_{\delta} \cap\left\{\tau_{\mathcal{\varepsilon}, \zeta}=\infty\right\}\right\}} \mathfrak{z}_{T} \leq 1$ and, in turn, $\mathrm{E} I_{\left\{\mathfrak{B}_{\delta} \cap\left\{\tau_{\varepsilon, \zeta}=\infty\right\}\right\}} \mathfrak{z}_{*} \leq 1$. The latter implies

$$
\begin{aligned}
\varlimsup_{\zeta \rightarrow 0} \varlimsup_{\delta \rightarrow 0} \varlimsup_{\varepsilon \rightarrow 0} \varepsilon^{2 \kappa} \log \mathrm{P}\left(\mathfrak{B}_{\delta} \cap\left\{\tau_{\varepsilon, \zeta=\infty}\right\}\right) & \\
& \leq-\sum_{i=1}^{n}\left[\lambda_{i}\left(\left[u_{t_{i}^{n}}-u_{s_{i}^{n}}\right]-\mathbf{b}\left[t_{i}^{n}-s_{i}^{n}\right]\right)-\frac{\lambda_{i}^{2}}{2} \mathbf{a}\left[t_{i}^{n}-s_{i}^{n}\right]\right]
\end{aligned}
$$

and it is left to choose $\lambda_{i}=\frac{\left[u_{t}^{n}-u_{\left.s_{i}^{n}\right]}\right]-\mathbf{b}\left[t_{i}^{n}-s_{i}^{n}\right]}{\mathbf{a}\left[t_{i}^{n}-s_{i}^{n}\right]}$ and apply (A.15).

(c.3) is obvious. 
A.2.3. Lower bound. Since the upper bound equals $-\infty$ for any $u \in \mathfrak{U}_{2}$, the lower bound has to be checked for $u \in \mathfrak{U}_{1}$ only.

Lemma A.3. A.9 holds with $J(u)=\frac{1}{2 \mathbf{a}} \int_{0}^{T}\left(\dot{u}_{t}-\mathbf{b}\right)^{2} d t$ for any $u \in \mathfrak{U}_{1}$.

Proof. It suffices to prove

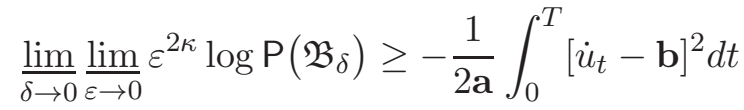

only for $u$ with continuous second derivative $\ddot{u}_{t}$. Indeed, as $\int_{0}^{T} \dot{u}_{t}^{2} d t<\infty$, there exists a sequence $\left(u_{m}(t)\right)_{m \geq 1}$ of twice continuously differentiable functions such that their derivatives $\left(\dot{u}_{m}(t)\right)_{m \geq 1}$ approximate $\dot{u}_{t}: \lim _{m \rightarrow \infty} \int_{0}^{T}\left(\dot{u}_{t}-\dot{u}_{m}(t)\right)^{2} d t=0$. The latter also provides

$$
\sup _{t \leq T}\left|u_{t}-u_{m}(t)\right| \leq\left(T \int_{0}^{T}\left[\dot{u}_{s}-\dot{u}_{m}(s)\right]^{2} d s\right)^{1 / 2} \underset{m \rightarrow \infty}{\longrightarrow} 0 .
$$

Set $\mathfrak{B}_{\delta, m}=\left\{\sup _{t \leq T}\left|X_{t}^{\varepsilon}-u_{m}(t)\right| \leq \delta\right\}$ and suppose we know that

$$
\varliminf_{\delta \rightarrow 0} \varliminf_{\varepsilon \rightarrow 0} \varepsilon^{2 \kappa} \log \mathrm{P}\left(\mathfrak{B}_{\delta, m}\right) \geq-\frac{1}{2 \mathbf{a}} \int_{0}^{T}\left[\dot{u}_{m}(t)-\mathbf{b}\right]^{2} d t .
$$

For $\gamma>\delta>0$, write

$$
\begin{aligned}
\mathfrak{B}_{\delta, m} & \subseteq\left(\mathfrak{B}_{\delta, m} \cap\left\{\sup _{t \leq T}\left|u_{t}-u_{m}(t)\right| \leq \gamma\right\}\right) \bigcup\left\{\sup _{t \leq T}\left|u_{t}-u_{m}(t)\right|>\gamma\right\} \\
& \subseteq\left\{\sup _{t \leq T}\left|X_{t}^{\varepsilon}-u_{t}\right| \leq 2 \gamma\right\} \bigcup\left\{\sup _{t \leq T}\left|u_{t}-u_{m}(t)\right|>\gamma\right\} .
\end{aligned}
$$

For sufficiently large $m$, the set $\left\{\sup _{t \leq T}\left|u_{t}-u_{m}(t)\right|>\gamma\right\}$ is empty.

Hence, $\mathrm{P}\left(\sup _{t \leq T}\left|X_{t}^{\varepsilon}-u_{t}\right| \leq 2 \gamma\right) \geq \mathrm{P}\left(\mathfrak{B}_{\delta, m}\right)$ and, therefore,

$$
\varliminf_{\varepsilon \rightarrow 0} \varepsilon^{2 \kappa} \log \mathrm{P}\left(\sup _{t \leq T}\left|X_{t}^{\varepsilon}-u_{t}\right| \leq 2 \gamma\right) \geq-\frac{1}{2 \mathbf{a}} \int_{0}^{T}\left[\dot{u}_{t}-\mathbf{b}\right]^{2} d t .
$$

and it is left to pass to the limit with $\gamma \rightarrow 0$.

The second helpful fact is that (A.17) with $u$, having continuously differentiable $\ddot{u}$, follows from

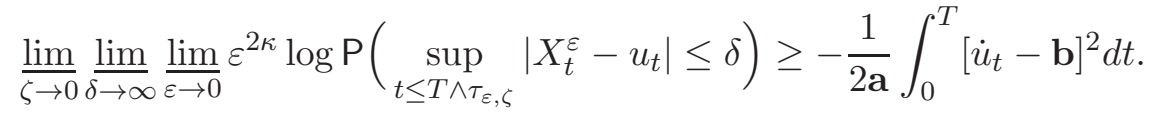

Write

$$
\begin{aligned}
\left\{\sup _{t \leq T \wedge \tau_{\varepsilon, \zeta}}\left|X_{t}^{\varepsilon}-u_{t}\right| \leq \delta\right\}= & \left\{\sup _{t \leq T}\left|X_{t}^{\varepsilon}-u_{t}\right| \leq \delta, \tau_{\varepsilon, \zeta}=\infty\right\} \\
& \bigcup\left\{\sup _{t \leq T \wedge \tau_{\varepsilon, \zeta}}\left|X_{t}^{\varepsilon}-u_{t}\right| \leq \delta, \tau_{\varepsilon, \zeta}<\infty\right\} \\
& \subseteq\left\{\sup _{t \leq T}\left|X_{t}^{\varepsilon}-u_{t}\right| \leq \delta\right\} \bigcup\left\{\tau_{\varepsilon, \zeta}<\infty\right\} .
\end{aligned}
$$


Hence, we obtain the following inequality

$$
\mathrm{P}\left(\sup _{t \leq T \wedge \tau_{\varepsilon, \zeta}}\left|X_{t}^{\varepsilon}-u_{t}\right| \leq \delta\right) \leq 2\left[\mathrm{P}\left(\sup _{t \leq T}\left|X_{t}^{\varepsilon}-u_{t}\right| \leq \delta\right) \bigvee \mathrm{P}\left(\tau_{\varepsilon, \zeta}<\infty\right)\right]
$$

and in view of (․19) and $\varlimsup_{\zeta \rightarrow \infty} \varlimsup_{\varepsilon \rightarrow 0} \varepsilon^{2 \kappa} \log \mathrm{P}\left(\tau_{\varepsilon, \zeta}<\infty\right)=-\infty$, we obtain the required (A.17).

Henceforth, we focus on the proof of (A.19), where $u_{t}$ is assumed to be twice continuously differentiable function.

Let us introduce a martingale exponential $\mathfrak{z} t=e^{M_{t}-\frac{1}{2}\langle M\rangle_{t}}$ with

$$
M_{t}=\frac{1}{\varepsilon^{\kappa}} \int_{0}^{t \wedge \tau_{\varepsilon, \zeta}} \frac{\dot{u}_{t}-\beta_{s}^{\varepsilon}}{\alpha_{s}^{\varepsilon}} d B_{s} \quad \text { and } \quad\langle M\rangle_{t}=\frac{1}{\varepsilon^{2 \kappa}} \int_{0}^{t \wedge \tau_{\varepsilon, \zeta}} \frac{\left(\dot{u}_{t}-\beta_{s}^{\varepsilon}\right)^{2}}{\left(\alpha_{t}^{\varepsilon}\right)^{2}} d t .
$$

Since $\dot{u}_{t}$ is bounded, $\langle M\rangle_{T}$ is bounded too. Hence, we have $\mathrm{E}_{\mathfrak{z} T}=1$. Set $d \overline{\mathrm{P}}=\mathfrak{z}_{T} d \mathrm{P}$. Owing to $\mathfrak{z}_{T}>0$, P- a.s., we have $\overline{\mathrm{P}} \sim \mathrm{P}$ with $d \mathrm{P}=\mathfrak{z}_{T}^{-1} d \overline{\mathrm{P}}$. Write

$$
\mathrm{P}\left(\sup _{t \leq T \wedge \tau_{\varepsilon, \zeta}}\left|X_{t}^{\varepsilon}-u_{t}\right| \leq \delta\right)=\int_{\left\{\sup _{t \leq T \wedge \tau_{\varepsilon, \zeta}}\left|X_{t}^{\varepsilon}-u_{t}\right| \leq \delta\right\}} \mathfrak{z}_{T}^{-1} d \overline{\mathrm{P}} .
$$

First we show that

$$
\varliminf_{\zeta \rightarrow 0} \varliminf_{\varepsilon \rightarrow 0} \overline{\mathrm{P}}\left(\sup _{t \leq T \wedge \tau_{\varepsilon, \zeta}}\left|X_{t}^{\varepsilon}-u_{t}\right| \leq \delta\right)=1 .
$$

By the Girsanov theorem (see, e.g., Theorem 2, $\S 5$, Ch. 4 in [10]), the random process

$$
\bar{B}_{t}=B_{t}-\frac{1}{\varepsilon^{\kappa}} \int_{0}^{t \wedge \tau_{\varepsilon, \zeta}} \frac{\dot{u}_{s}-\beta_{s}^{\varepsilon}}{\alpha_{s}^{\varepsilon}} d s
$$

is a Brownian motion under $\overline{\mathrm{P}}$. Both process $X_{t}^{\varepsilon}$ and $\mathfrak{z} t$ are semimartingales under $\overline{\mathrm{P}}$. In particular,

$$
X_{t \wedge \tau_{\varepsilon, \zeta}}^{\varepsilon}=x_{0}+\int_{0}^{t \wedge \tau_{\varepsilon, \zeta}} \dot{u}_{s} d s+\varepsilon^{\kappa} \int_{0}^{t \wedge \tau_{\varepsilon, \zeta}} \alpha_{s}^{\varepsilon} d \bar{B}_{s}=u_{t \wedge \tau_{\varepsilon, \zeta}}+\varepsilon^{\kappa} \int_{0}^{t \wedge \tau_{\varepsilon, \zeta}} \alpha_{s}^{\varepsilon} d \bar{B}_{s} .
$$

Consequently,

$$
\sup _{t \leq T \wedge \tau_{\varepsilon, \zeta}}\left|X_{t}^{\varepsilon}-u_{t}\right|=\sup _{t \leq T}\left|\varepsilon^{\kappa} \int_{0}^{t \wedge \tau_{\varepsilon, \zeta}} \alpha_{s}^{\varepsilon} d \bar{B}_{s}\right|
$$

Using the Doob inequality, we find that

$$
\begin{aligned}
& \overline{\mathrm{P}}\left(\sup _{t \leq T \wedge \tau_{\varepsilon, \zeta}}\left|X_{t}^{\varepsilon}-u_{t}\right| \leq \delta\right)=\overline{\mathrm{P}}\left(\sup _{t \leq T}\left|\varepsilon^{\kappa} \int_{0}^{t \wedge \tau_{\varepsilon, \zeta}} \alpha_{s}^{\varepsilon} d \bar{B}_{s}\right| \leq \delta\right) \\
& =1-\overline{\mathrm{P}}\left(\sup _{t \leq T}\left|\varepsilon^{\kappa} \int_{0}^{t \wedge \tau_{\varepsilon, \zeta}} \alpha_{s}^{\varepsilon} d \bar{B}_{s}\right|>\delta\right) \\
& \geq 1-\frac{4 \varepsilon^{2 \kappa}}{\delta^{2}} \overline{\mathrm{E}} \int_{0}^{T \wedge \tau_{\varepsilon, \zeta}}\left(\alpha_{s}^{\varepsilon}\right)^{2} d s \geq 1-\frac{4 \varepsilon^{2 \kappa}}{\delta^{2}}[\mathbf{a} T+\zeta] \underset{\varepsilon \rightarrow 0}{\longrightarrow} 1 .
\end{aligned}
$$


We return to the proof of (A.19). Due to (A.22), we have

$$
\mathfrak{z}_{T}=\exp \left(\frac{1}{\varepsilon^{\kappa}} \int_{0}^{T \wedge \tau_{\varepsilon, \zeta}} \frac{\dot{u}_{t}-\beta_{s}^{\varepsilon}}{\alpha_{s}^{\varepsilon}} d \bar{B}_{s}+\frac{1}{2 \varepsilon^{2 \kappa}} \int_{0}^{T \wedge \tau_{\varepsilon, \zeta}} \frac{\left[\dot{u}_{t}-\beta_{s}^{\varepsilon}\right]^{2}}{\left(\alpha_{s}^{\varepsilon}\right)^{2}} d s\right)
$$

and, setting $\mathfrak{B}_{\delta, \varepsilon}:=\left\{\sup _{t \leq T \wedge \tau_{\varepsilon, \zeta}}\left|X_{t}^{\varepsilon}-u_{t}\right| \leq \delta\right\}$, transform A.20) to

$$
\mathrm{P}\left(\mathfrak{B}_{\delta, \varepsilon}\right)=\int_{\mathfrak{B}_{\delta, \varepsilon}} \exp \left(-\frac{1}{\varepsilon^{2 \kappa}} \int_{0}^{T \wedge \tau_{\varepsilon, \zeta}} \frac{\dot{u}_{t}-\beta_{s}^{\varepsilon}}{\alpha_{s}^{\varepsilon}} d \bar{B}_{s}-\frac{1}{2 \varepsilon^{2 \kappa}} \int_{0}^{T \wedge \tau_{\varepsilon, \zeta}} \frac{\left[\dot{u}_{s}-\beta_{s}^{\varepsilon}\right]^{2}}{\left(\alpha_{s}^{\varepsilon}\right)^{2}} d s\right) d \overline{\mathrm{P}} .
$$

Further, it is convenient to apply a few obvious relations. We choose $l$ as un upper bound for

$$
\frac{\left(\dot{u}_{s}-\mathbf{b}\right)^{2}}{\mathbf{a}\left(\alpha_{s}^{\varepsilon}\right)^{2}}+\frac{2\left|\dot{u}_{s}-\mathbf{b}\right|+\left|\mathbf{b}-\beta_{s}^{\varepsilon}\right|}{\mathbf{a}}\left[1+\frac{\left|\mathbf{a}-\left(\alpha_{s}^{\varepsilon}\right)^{2}\right|}{\left(\alpha_{s}^{\varepsilon}\right)^{2}}\right] .
$$

Write

$$
\begin{aligned}
\int_{0}^{T \wedge \tau_{\varepsilon, \zeta}} \frac{\left[\dot{u}_{s}-\beta_{s}^{\varepsilon}\right]^{2}}{\left(\alpha_{s}^{\varepsilon}\right)^{2}} d s= & \int_{0}^{T \wedge \tau_{\varepsilon, \zeta}} \frac{\left[\left(\dot{u}_{s}-\mathbf{b}\right)+\left(\mathbf{b}-\beta_{s}^{\varepsilon}\right)\right]^{2}}{\mathbf{a}}\left[1+\frac{\mathbf{a}-\left(\alpha_{s}^{\varepsilon}\right)^{2}}{\left(\alpha_{s}^{\varepsilon}\right)^{2}}\right] d s \\
\leq & \int_{0}^{T \wedge \tau_{\varepsilon, \zeta}} \frac{\left(\dot{u}_{s}-\mathbf{b}\right)^{\mathbf{2}}}{\mathbf{a}} d s+\int_{0}^{T \wedge \tau_{\varepsilon, \zeta}} \frac{\left(\dot{u}_{s}-\mathbf{b}\right)^{2}}{\mathbf{a}-\left(\alpha_{s}^{\varepsilon}\right)^{2} \mid} \frac{\left.\mid \alpha_{s}^{\varepsilon}\right)^{2}}{\mathbf{a}} d s \\
& +\int_{0}^{T \wedge \tau_{\varepsilon, \zeta}} \frac{2\left|\dot{u}_{s}-\mathbf{b}\right|\left|\mathbf{b}-\beta_{s}^{\varepsilon}\right|+\left(\mathbf{b}-\beta_{s}^{\varepsilon}\right)^{2}}{\mathbf{a}}\left[1+\frac{\left|\mathbf{a}-\left(\alpha_{s}^{\varepsilon}\right)^{2}\right|}{\left(\alpha_{s}^{\varepsilon}\right)^{2}}\right] d s \\
\leq & \int_{0}^{T \wedge \tau_{\varepsilon, \zeta}} \frac{\left(\dot{u}_{s}-\mathbf{b}\right)^{\mathbf{2}}}{\mathbf{a}} d s+l \int_{0}^{T \wedge \tau_{\varepsilon, \zeta}}\left[\left|\mathbf{a}-\left(\alpha_{s}^{\varepsilon}\right)^{2}\right|+\left|\mathbf{b}-\beta_{s}^{\varepsilon}\right|\right] d s \\
\leq & \int_{0}^{T \wedge \tau_{\varepsilon, \zeta}} \frac{\left(\dot{u}_{s}-\mathbf{b}\right)^{\mathbf{2}}}{\mathbf{a}} d s+\zeta l .
\end{aligned}
$$

The latter implies

$$
\begin{aligned}
& \int_{\mathfrak{B}_{\delta, \varepsilon}} \exp \left(-\frac{1}{\varepsilon^{\kappa}} \int_{0}^{T \wedge \tau_{\varepsilon, \zeta}} \frac{\dot{u}_{s}-\beta_{s}^{\varepsilon}}{\alpha_{s}^{\varepsilon}} d \bar{B}_{s}-\frac{1}{2 \varepsilon^{2 \kappa}} \int_{0}^{T \wedge \tau_{\varepsilon, \zeta}} \frac{\left[\dot{u}_{s}-\beta_{s}^{\varepsilon}\right]^{2}}{\left(\alpha_{s}^{\varepsilon}\right)^{2}} d s\right) d \overline{\mathrm{P}} \\
& \geq \exp \left(-\frac{1}{2 \varepsilon^{2 \kappa}} \int_{0}^{T} \frac{\left(\dot{u}_{s}-\mathbf{b}\right)^{2}}{\mathbf{a}} d s-\frac{\zeta l}{2 \varepsilon^{2 \kappa}}\right) \int_{\mathfrak{B}_{\delta, \zeta}} 1 \wedge \exp \left(-\frac{1}{\varepsilon^{\kappa}} \int_{0}^{T \wedge \tau_{\varepsilon, \zeta}} \frac{\dot{u}_{t}-\beta_{s}^{\varepsilon}}{\left(\alpha_{s}^{\varepsilon}\right)^{2}} d \bar{B}\right) d \overline{\mathrm{P}}
\end{aligned}
$$

(" $1 \wedge \exp (\cdots)$ " is introduced in order to have bounded integrand in the last integral above). Thus, we obtain the following lower bound:

$$
\begin{aligned}
\mathrm{P}^{\varepsilon^{2 \kappa}}\left(\mathfrak{B}_{\delta, \varepsilon}\right) \geq \exp \left(-\frac{1}{2 \mathbf{a}} \int_{0}^{T}\left(\dot{u}_{s}-\right.\right. & \left.\mathbf{b})^{2} d t-\frac{\zeta l}{2}\right) \\
\times\left(\int_{\mathfrak{B}_{\delta, \varepsilon}} 1\right. & \left.\wedge \exp \left(-\frac{1}{\varepsilon^{\kappa}} \int_{0}^{T \wedge \tau_{\varepsilon, \zeta}} \frac{\dot{u}_{s}-\beta_{s}^{\varepsilon}}{\left(\alpha_{s}^{\varepsilon}\right)^{2}} d \bar{B}\right) d \overline{\mathrm{P}}\right)^{\varepsilon^{2 \kappa}} .
\end{aligned}
$$


Further, by the Hölder inequality (here $\varepsilon<1$ )

$$
\begin{aligned}
& \int_{\mathfrak{B}_{\delta, \varepsilon}} 1 \wedge \exp \left(-\varepsilon^{\kappa} \int_{0}^{T \wedge \tau_{\varepsilon, \zeta}} \frac{\dot{u}_{s}-\beta_{s}^{\varepsilon}}{\left(\alpha_{s}^{\varepsilon}\right)^{2}} d \bar{B}_{s}\right) d \overline{\mathrm{P}} \\
& \leq\left(\int_{\mathfrak{B}_{\delta, \varepsilon}} 1 \wedge \exp \left(-\frac{1}{\varepsilon^{\kappa}} \int_{0}^{T \wedge \tau_{\varepsilon, \zeta}} \frac{\dot{u}_{s}-\beta_{s}^{\varepsilon}}{\left(\alpha_{s}^{\varepsilon}\right)^{2}} d \bar{B}_{s}\right) d \overline{\mathrm{P}}\right)^{\varepsilon^{2 \kappa}} .
\end{aligned}
$$

Thus, we obtain

$$
\begin{aligned}
& \varliminf_{\varepsilon \rightarrow 0} \varepsilon^{2 \kappa} \log \mathrm{P}\left(\sup _{t \leq T \wedge \tau_{\varepsilon, \zeta}}\left|X_{t}^{\varepsilon}-u_{t}\right| \leq \delta\right) \geq-\frac{1}{2 \mathbf{a}} \int_{0}^{T}\left(\dot{u}_{s}-\mathbf{b}\right)^{2} d s \\
& -\frac{\zeta l}{2}+\varliminf_{\varepsilon \rightarrow 0} \varepsilon^{2 \kappa} \log \int_{\mathfrak{B}_{\delta, \varepsilon}} 1 \wedge \exp \left(-\varepsilon^{\kappa} \int_{0}^{T \wedge \tau_{\varepsilon, \zeta}} \frac{\dot{u}_{s}-\beta_{s}^{\varepsilon}}{\left(\alpha_{s}^{\varepsilon}\right)^{2}} d \bar{B}_{s}\right) d \overline{\mathrm{P}} \\
& \geq-\frac{1}{2 \mathbf{a}} \int_{0}^{T}\left(\dot{u}_{s}-\mathbf{b}\right)^{2} d s-\frac{\zeta l}{2}+\log \frac{\lim }{\varepsilon \rightarrow 0} \int_{\mathfrak{B}_{\delta, \varepsilon}} \psi^{\varepsilon} d \overline{\mathrm{P}},
\end{aligned}
$$

where $\psi^{\varepsilon}:=1 \wedge \exp \left(-\varepsilon^{\kappa} \int_{0}^{T \wedge \tau_{\varepsilon, \zeta}} \frac{\dot{u}_{s}-\beta_{s}^{\varepsilon}}{\left(\alpha_{s}^{\varepsilon}\right)^{2}} d \bar{B}_{s}\right)$. In view of $(\underline{\mathrm{A} .23}), \lim _{\varepsilon \rightarrow 0} \overline{\mathrm{P}}\left(\mathfrak{B}_{\delta, \varepsilon}\right)=1$ and, also, $\lim _{\varepsilon \rightarrow 0} \int_{\Omega} \psi^{\varepsilon} d \overline{\mathrm{P}}=1$. Hence, owing to $\psi^{\varepsilon} \leq 1$,

$$
\begin{aligned}
\int_{\mathfrak{B}_{\delta, \varepsilon}} \psi^{\varepsilon} d \overline{\mathrm{P}} & =\int_{\Omega} \psi^{\varepsilon} d \overline{\mathrm{P}}-\int_{\Omega \backslash \mathfrak{B}_{\delta, \varepsilon}} \psi^{\varepsilon} d \overline{\mathrm{P}} \\
& \geq \int_{\Omega} \psi^{\varepsilon} d \overline{\mathrm{P}}-\int_{\Omega \backslash \mathfrak{B}_{\delta, \varepsilon}} d \overline{\mathrm{P}} \underset{\varepsilon \rightarrow 0}{\longrightarrow} 1,
\end{aligned}
$$

that is, $\log \underline{\lim }_{\varepsilon \rightarrow 0} \int_{\mathfrak{B}_{\delta, \varepsilon}} \psi^{\varepsilon} d \overline{\mathrm{P}} \geq 0$.

Consequently, by an arbitrariness of $\zeta$, (A.17) is valid.

\section{REFERENCES}

[1] Brox, Th. A one-dimensional diffusion process in a Wiener medium. Ann. Probab. 14 (1986), no. 4, $1206-1218$

[2] Dembo, A. and Zeitouni, O. Large deviations techniques and applications. Jones and Bartlett Publishers, Boston, MA, 1993.

[3] Dupuis, P., Ellis, R. (1997) A Weak Convergence Approach to the Theory of Large Deviations. Wiley.

[4] Gärtner, J. and Freidlin, M.I., (1979) On the propagation of concentration waves in periodic and random media. Dokl. Acad. Nauk SSSR 249, 521-525 (in Russian).

[5] Freidlin, M. and Sowers, R. (1999) A comparison of homogenization and large deviations, with applications to wavefront propagation. Stochastic Processes and their Applications. 82, 23-52

[6] Freidlin, M.I., Wentzell A.D. (1984)Random Perturbations of Dynamical Systems. N.Y. Springer.

[7] Krylov, N. V. Controlled diffusion processes. Applications of Mathematics, 14. Springer-Verlag, New York-Berlin, 1980

[8] Jacod, J. and Shiryaev, A.N. Limit theorems for stochastic processes. Second edition. Fundamental Principles of Mathematical Sciences, 288. Springer-Verlag, Berlin, 2003

[9] Liptser, R.S. and Shiryaev, A.N. Statistics of random processes. I. General theory. Second edition. Applications of Mathematics (New York), 5. Stochastic Modelling and Applied Probability. Springer-Verlag, Berlin, 2001 
[10] Liptser, R. Sh.; Shiryayev, A. N. Theory of martingales. Mathematics and its Applications (Soviet Series), 49. Kluwer Academic Publishers Group, Dordrecht, 1989

[11] Liptser, R.; Spokoiny, V. Moderate deviations type evaluation for integral functionals of diffusion processes. Electron. J. Probab. 4 (1999), no. 17, 25 pp

[12] Liptser, R.S. and Pukhalskii, A.A. (1992) Limit theorems on large deviations for semimartingales, Stochastics and Stochastic Reports. 38, pp. 201-249.

[13] Puhalskii, A.A. Large deviations and idempotent probability. Chapman \& Hall/CRC Monographs and Surveys in Pure and Applied Mathematics, 119. Chapman \& Hall/CRC, Boca Raton, FL, 2001

[14] Puhalskii, A.A. Large deviations of semimartingales via convergence of the predictable characteristics, Stoch. Stoch. Rep., v. 49(1994), pp. 27-85

[15] Schumacher, S. Diffusions with random coefficients. Particle systems, random media and large deviations (Brunswick, Maine, 1984), 351-356, Contemp. Math., 41, Amer. Math. Soc., Providence, RI, 1985.

Universite du Maine, Faculte des Sciences, Laboratoire de Statistique et Processus, Avenue Olivier Messiaen, 72085 Le Mans CEDEX 9

E-mail address: Pavel.Chigansky@univ-lemans.fr

Department of Electrical Engineering Systems, Tel Aviv University, 69978 Tel Aviv, Israel

E-mail address: liptser@eng.tau.ac.il 